\title{
Separation of contributions from deeply virtual Compton scattering and its interference with the Bethe-Heitler process in measurements on a hydrogen target
}

\section{The HERMES Collaboration}

ABSTRACT: Hard exclusive leptoproduction of real photons from an unpolarized proton target is studied in an effort to elucidate generalized parton distributions. The data accumulated during the years 1996-2005 with the HERMES spectrometer are analyzed to yield asymmetries with respect to the combined dependence of the cross section on beam helicity and charge, thereby revealing previously unseparated contributions from deeply virtual Compton scattering and its interference with the Bethe-Heitler process. The integrated luminosity is sufficient to show correlated dependences on two kinematic variables, and provides the most precise determination of the dependence on only the beam charge.

KEYwORDs: Lepton-Nucleon Scattering. 


\section{The HERMES Collaboration}

A. Airapetian ${ }^{12,15}$, N. Akopov ${ }^{26}$, Z. Akopov ${ }^{5}$, E.C. Aschenauer ${ }^{6, a}$, W. Augustyniak ${ }^{25}$, R. Avakian ${ }^{26}$, A. Avetissian ${ }^{26}$, E. Avetisyan ${ }^{5}$, B. Ball ${ }^{15}$, S. Belostotski ${ }^{18}$, H.P. Blok ${ }^{17,24}$, A. Borissov ${ }^{5}$, J. Bowles ${ }^{13}$, V. Bryzgalov ${ }^{19}$, J. Burns ${ }^{13}$, G.P. Capitani ${ }^{10}$, E. Cisbani ${ }^{21}$, G. Ciullo ${ }^{9}$, M. Contalbrigo ${ }^{9}$, P.F. Dalpiaz ${ }^{9}$, W. Deconinck ${ }^{5,15, b}$, R. De Leo ${ }^{2}$, L. De Nardo ${ }^{15,5}$, E. De Sanctis ${ }^{10}$, M. Diefenthaler ${ }^{14,8}$, P. Di Nezza ${ }^{10}$, M. Düren ${ }^{12}$, M. Ehrenfried ${ }^{12, c}$, G. Elbakian ${ }^{26}$, F. Ellinghaus ${ }^{4, d}$, R. Fabbri ${ }^{6}$, A. Fantoni ${ }^{10}$, L. Felawka ${ }^{22}$, S. Frullani ${ }^{21}$, D. Gabbert ${ }^{6}$, G. Gapienko ${ }^{19}$, V. Gapienko ${ }^{19}$, F. Garibaldi ${ }^{21}$, G. Gavrilov ${ }^{5,18,22}$, V. Gharibyan ${ }^{26}$, F. Giordano ${ }^{5,9}$, S. Gliske ${ }^{15}$, C. Hadjidakis ${ }^{10, e}$, M. Hartig ${ }^{5, f}$, D. Hasch ${ }^{10}$, G. Hill ${ }^{13}$, A. Hillenbrand ${ }^{6}$, M. Hoek ${ }^{13}$, Y. Holler ${ }^{5}$, I. Hristova ${ }^{6}$, Y. Imazu ${ }^{23}$, A. Ivanilov ${ }^{19}$, H.E. Jackson ${ }^{1}$, H.S. Jo ${ }^{11}$, S. Joosten ${ }^{14,11}$, R. Kaiser ${ }^{13}$, G. Karyan ${ }^{26}$, T. Keri ${ }^{13,12}$, E. Kinney ${ }^{4}$, A. Kisselev ${ }^{18}$, N. Kobayashi ${ }^{23}$, V. Korotkov ${ }^{19}$, V. Kozlov ${ }^{16}$, P. Kravchenko ${ }^{18}$, L. Lagamba ${ }^{2}$, R. Lamb ${ }^{14}$, L. Lapikás ${ }^{17}$, I. Lehmann ${ }^{13}$, P. Lenisa ${ }^{9}$, A. López Ruiz ${ }^{11}$, W. Lorenzon $^{15}$, X.-G. Lu ${ }^{6}$, X.-R. $\mathbf{L u}^{23, g}$, B.-Q. $\mathbf{M a}^{3}$, D. Mahon ${ }^{13}$, N.C.R. Makins ${ }^{14}$, S.I. Manaenkov ${ }^{18}$, Y. $\mathrm{Mao}^{3}$, B. Marianski ${ }^{25}$, A. Martinez de la Ossa ${ }^{4}$, H. Marukyan ${ }^{26}$, C.A. Miller $^{22}$, Y. Miyachi ${ }^{23}$, A. Movsisyan ${ }^{26}$, V. Muccifora ${ }^{10}$, M. Murray ${ }^{13}$, A. Mussgiller ${ }^{5,8}$, E. Nappi ${ }^{2}$, Y. Naryshkin ${ }^{18}$, A. Nass $^{8}$, M. Negodaev ${ }^{6}$, W.-D. Nowak $^{6}$, L.L. Pappalardo ${ }^{9}$, R. Perez-Benito ${ }^{12}$, N. Pickert ${ }^{8, h}$, M. Raithel ${ }^{8}$, P.E. Reimer ${ }^{1}$, A.R. Reolon ${ }^{10}$, C. Riedl ${ }^{6}$, K. Rith ${ }^{8}$, G. Rosner ${ }^{13}$, A. Rostomyan ${ }^{5}$, J. Rubin ${ }^{14}$, D. Ryckbosch ${ }^{11}$, Y. Salomatin ${ }^{19}$, F. Sanft ${ }^{20}$,

A. Schäfer ${ }^{20}$, G. Schnell ${ }^{6,11}$, K.P. Schüler ${ }^{5}$, B. Seitz ${ }^{13}$, T.-A. Shibata ${ }^{23}$, V. Shutov ${ }^{7}$, M. Stancari ${ }^{9}$, M. Statera ${ }^{9}$, E. Steffens ${ }^{8}$, J.J.M. Steijger ${ }^{17}$, H. Stenzel ${ }^{12}$, J. Stewart ${ }^{6, a}$, F. Stinzing ${ }^{8}$, A. Terkulov ${ }^{16}$, A. Trzcinski ${ }^{25}$, M. Tytgat ${ }^{11}$, Y. Van Haarlem ${ }^{11, i}$, C. Van Hulse ${ }^{11}$, D. Veretennikov ${ }^{18}$,

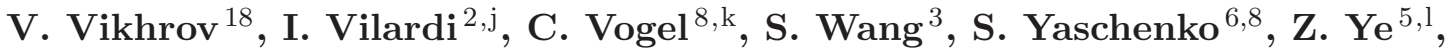
S. Yen ${ }^{22}$, W. Yu ${ }^{12}$, D. Zeiler ${ }^{8}$, B. Zihlmann ${ }^{5, m}$, P. Zupranski ${ }^{25}$

\footnotetext{
${ }^{a}$ Now at: Brookhaven National Laboratory, Upton, New York 11772-5000, USA

${ }^{\mathrm{b}}$ Now at: Massachusetts Institute of Technology, Cambridge, Massachusetts 02139, USA

${ }^{\mathrm{c}}$ Now at: Siemens AG Molecular Imaging, 91052 Erlangen, Germany

${ }^{\mathrm{d} N o w}$ at: Institut für Physik, Universität Mainz, 55128 Mainz, Germany

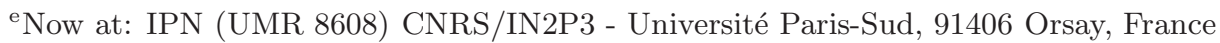

${ }^{\mathrm{f}}$ Now at: Institut für Kernphysik, Universität Frankfurt a.M., 60438 Frankfurt a.M., Germany

${ }^{\mathrm{g}}$ Now at: Graduate University of Chinese Academy of Sciences, Beijing 100049, China

${ }^{\mathrm{h}}$ Now at: Siemens AG, 91301 Forchheim, Germany

${ }^{\mathrm{i}}$ Now at: Carnegie Mellon University, Pittsburgh, Pennsylvania 15213, USA

${ }^{j}$ Now at: IRCCS Multimedica Holding S.p.A., 20099 Sesto San Giovanni (MI), Italy

${ }^{\mathrm{k}}$ Now at: AREVA NP GmbH, 91058 Erlangen, Germany

${ }^{1}$ Now at: Fermi National Accelerator Laboratory, Batavia, Illinois 60510, USA

${ }^{\mathrm{m}}$ Now at: Thomas Jefferson National Accelerator Facility, Newport News, Virginia 23606, USA
} 
1 Physics Division, Argonne National Laboratory, Argonne, Illinois 60439-4843, USA

2 Istituto Nazionale di Fisica Nucleare, Sezione di Bari, 70124 Bari, Italy

3 School of Physics, Peking University, Beijing 100871, China

4 Nuclear Physics Laboratory, University of Colorado, Boulder, Colorado 80309-0390, $U S A$

5 DESY, 22603 Hamburg, Germany

6 DESY, 15738 Zeuthen, Germany

7 Joint Institute for Nuclear Research, 141980 Dubna, Russia

8 Physikalisches Institut, Universität Erlangen-Nürnberg, 91058 Erlangen, Germany

9 Istituto Nazionale di Fisica Nucleare, Sezione di Ferrara and Dipartimento di Fisica, Università di Ferrara, 44100 Ferrara, Italy

10 Istituto Nazionale di Fisica Nucleare, Laboratori Nazionali di Frascati, 00044 Frascati, Italy

11 Department of Subatomic and Radiation Physics, University of Gent, 9000 Gent, Belgium

12 Physikalisches Institut, Universität Gießen, 35392 Gießen, Germany

13 Department of Physics and Astronomy, University of Glasgow, Glasgow G12 8QQ, United Kingdom

14 Department of Physics, University of Illinois, Urbana, Illinois 61801-3080, USA

15 Randall Laboratory of Physics, University of Michigan, Ann Arbor, Michigan 48109-1040, USA

16 Lebedev Physical Institute, 117924 Moscow, Russia

17 National Institute for Subatomic Physics (Nikhef), 1009 DB Amsterdam, The Netherlands

18 Petersburg Nuclear Physics Institute, Gatchina, Leningrad region 188300, Russia

19 Institute for High Energy Physics, Protvino, Moscow region 142281, Russia

20 Institut für Theoretische Physik, Universität Regensburg, 93040 Regensburg, Germany

21 Istituto Nazionale di Fisica Nucleare, Sezione Roma 1, Gruppo Sanità and Physics Laboratory, Istituto Superiore di Sanità, 00161 Roma, Italy

22 TRIUMF, Vancouver, British Columbia V6T 2A3, Canada

23 Department of Physics, Tokyo Institute of Technology, Tokyo 152, Japan

24 Department of Physics, VU University, $1081 \mathrm{HV}$ Amsterdam, The Netherlands

25 Andrzej Soltan Institute for Nuclear Studies, 00-689 Warsaw, Poland

26 Yerevan Physics Institute, 375036 Yerevan, Armenia 


\section{Introduction}

Parton Distribution Functions (PDFs) describe the longitudinal-momentum structure of the nucleon in the interpretation of inclusive and semi-inclusive Deep-Inelastic Scattering (DIS). Analogously, Generalized Parton Distributions (GPDs) [1, 2, 3] describe the multidimensional structure of the nucleon in the interpretation of hard exclusive leptoproduction, most simply when the target is left intact. PDFs and elastic nucleon Form Factors (FFs) are embodied in GPDs as their limiting cases and moments, respectively [2]. While FFs and PDFs represent one-dimensional distributions, GPDs provide correlated information on transverse spatial and longitudinal momentum distributions of partons [ 田, 5, 6]. In addition, the total angular momentum carried by partons in the nucleon can be calculated from GPDs [2].

GPDs depend on the four kinematic variables $x, \xi, Q^{2}$, and the squared four-momentum transfer $t$ to the target. In a frame where the nucleon moves with 'infinite' momentum, $x$ and $2 \xi$ are the average and difference of the longitudinal momentum fractions of the parton in the initial and final state, as illustrated in Fig. 1(a). In hard exclusive leptoproduction, $x$ has no direct relationship with the experimental kinematic observable $x_{\mathrm{B}} \equiv Q^{2} /(2 P \cdot q)$. Here, $P$ is the four momentum of the target nucleon, $q$ is the difference between the four momenta of the incident and scattered lepton, and $Q^{2} \equiv-q^{2}$. The skewness parameter $\xi$ is related to $x_{\mathrm{B}}$, as $\xi \approx x_{\mathrm{B}} /\left(2-x_{\mathrm{B}}\right)$ in leading order Quantum Electrodynamics (QED) and in the kinematic limit of large $Q^{2}$ with $x_{\mathrm{B}}$ and $t$ fixed (generalized Bjorken limit). In addition, like PDFs, GPDs are subject to Quantum Chromodynamics (QCD) evolution with $Q^{2}$, which has been calculated perturbatively to leading order [1, 2, 3, 7] and next-to-leading order [8, 9, 10] in the strong coupling constant $\alpha_{\mathrm{s}}$.

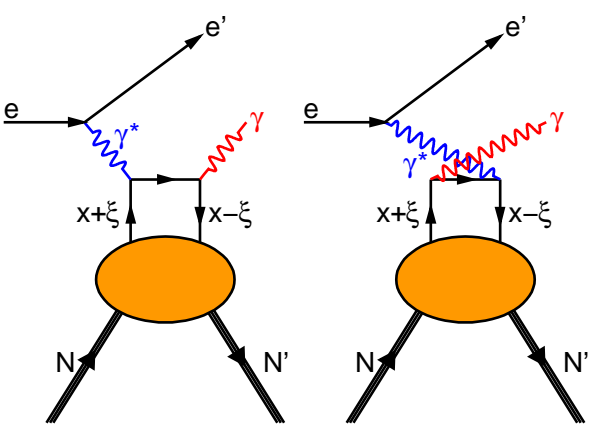

(a)

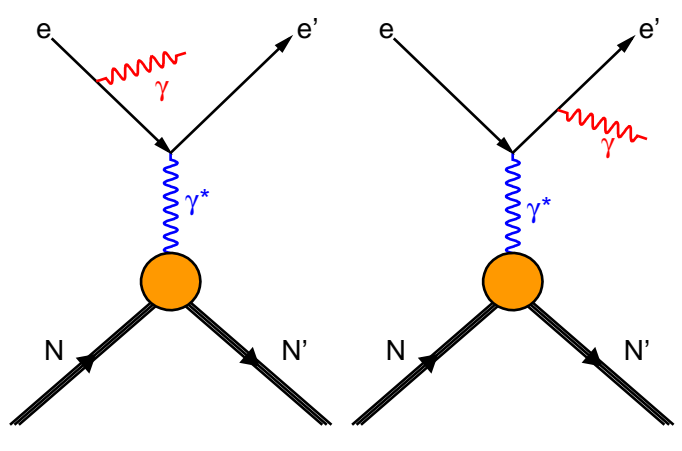

(b)

Figure 1: Leading order diagrams for deeply virtual Compton scattering (a) and Bethe-Heitler (b) processes.

Deeply Virtual Compton Scattering (DVCS), the hard exclusive leptoproduction of real photons, e.g., $e^{ \pm} p \rightarrow e^{ \pm} p \gamma$, has the simplest theoretical interpretation in terms of GPDs among the presently experimentally feasible hard exclusive reactions. DVCS amplitudes can be measured through the interference between the DVCS and Bethe-Heitler (BH) processes, in which the photon is radiated from a parton in the former and from the lepton in the latter (see Fig. 1). These processes have an identical final state. Hence their 
amplitudes $\tau_{\text {DVCS }}$ and $\tau_{\mathrm{BH}}$ add coherently, resulting in an interference term ' $\mathrm{I}$ ' in the cross section for exclusive leptoproduction of real photons. For an unpolarized proton target, the cross section can be written as [11, 12]

$$
\frac{\mathrm{d} \sigma}{\mathrm{d} x_{\mathrm{B}} \mathrm{d} Q^{2} \mathrm{~d}|t| \mathrm{d} \phi}=\frac{x_{\mathrm{B}} e^{6}}{32(2 \pi)^{4} Q^{4} \sqrt{1+\epsilon^{2}}}[\left|\tau_{\mathrm{BH}}\right|^{2}+\left|\tau_{\mathrm{DVCS}}\right|^{2}+\overbrace{\tau_{\mathrm{DVCS}} \tau_{\mathrm{BH}}^{*}+\tau_{\mathrm{DVCS}}^{*} \tau_{\mathrm{BH}}}^{\mathrm{I}}],
$$

where $e$ represents the elementary charge and $\epsilon \equiv 2 x_{\mathrm{B}} M_{p} / Q$, with $M_{p}$ the proton mass. The azimuthal angle $\phi$ is defined as the angle between the lepton scattering plane and the photon production plane spanned by the trajectories of the virtual and real photons.

The three contributions entering the photon production cross section can be expanded in Fourier series in $\phi$. For an unpolarized proton target, they can be written as 12

$$
\begin{aligned}
\left|\tau_{\mathrm{BH}}\right|^{2} & =\frac{K_{\mathrm{BH}}}{\mathcal{P}_{1}(\phi) \mathcal{P}_{2}(\phi)}\left\{\sum_{n=0}^{2} c_{n}^{\mathrm{BH}} \cos (n \phi)\right\}, \quad \text { with } \quad K_{\mathrm{BH}}=\frac{1}{x_{\mathrm{B}}^{2} t\left(1+\epsilon^{2}\right)^{2}}, \\
\left|\tau_{\mathrm{DVCS}}\right|^{2} & =\frac{1}{Q^{2}}\left\{\sum_{n=0}^{2} c_{n}^{\mathrm{DVCS}} \cos (n \phi)+\lambda s_{1}^{\mathrm{DVCS}} \sin \phi\right\} \\
\mathrm{I} & =\frac{-e_{\ell} K_{\mathrm{I}}}{\mathcal{P}_{1}(\phi) \mathcal{P}_{2}(\phi)}\left\{\sum_{n=0}^{3} c_{n}^{\mathrm{I}} \cos (n \phi)+\sum_{n=1}^{2} \lambda s_{n}^{\mathrm{I}} \sin (n \phi)\right\}, \quad \text { with } \quad K_{\mathrm{I}}=\frac{1}{x_{\mathrm{B}} y t}
\end{aligned}
$$

Here, $y$ is the fraction of the incident lepton energy carried by the virtual photon in the target rest frame, and $\lambda$ and $e_{\ell}$ represent respectively the beam helicity and beam charge in units of the elementary charge. The Fourier coefficients $c_{n}^{\mathrm{BH}}$ and lepton propagators $\mathcal{P}_{1}(\phi)$, $\mathcal{P}_{2}(\phi)$ of the $\mathrm{BH}$ term can be calculated within the framework of QED from the kinematic variables and the Dirac and Pauli form factors $F_{1}$ and $F_{2}$ of the nucleon.

The Fourier coefficients of the interference term (Eq. 1.4) are of greatest interest since they ultimately depend on a linear combination of GPDs, while the coefficients of the squared DVCS term (Eq. 1.3) are bilinear in GPDs. The coefficients

$$
\begin{aligned}
& s_{1}^{\mathrm{I}}=8 k \lambda y(2-y) \operatorname{Im} M^{1,1}, \\
& c_{1}^{\mathrm{I}}=8 k\left(2-2 y+y^{2}\right) \operatorname{Re} M^{1,1},
\end{aligned}
$$

are respectively proportional to the imaginary and real parts of $M^{1,1}$, the leading-twist (twist-2) photon-helicity-conserving amplitude of the DVCS process. Here, $M^{\mu, \mu^{\prime}}$ denotes the helicity amplitude with virtual (real) photon helicity $\mu\left(\mu^{\prime}\right)$, following the notation of Ref. [11]. The kinematic factor $k \propto \sqrt{-t} / Q$ originates from the BH propagators. Note that the sign in Eq. 1.6 differs from that in Ref. [12] due to the different definition of the azimuthal angle: $\phi=\pi-\phi_{[12}$. The amplitude $M^{1,1}$ is given by a linear combination of the Compton Form Factors $(\mathrm{CFFs}) \mathcal{H}, \widetilde{\mathcal{H}}$ and $\mathcal{E}$ :

$$
M^{1,1}=F_{1}(t) \mathcal{H}\left(\xi, t, Q^{2}\right)+\frac{x_{\mathrm{B}}}{2-x_{\mathrm{B}}}\left(F_{1}(t)+F_{2}(t)\right) \widetilde{\mathcal{H}}\left(\xi, t, Q^{2}\right)-\frac{t}{4 M_{p}^{2}} F_{2}(t) \mathcal{E}\left(\xi, t, Q^{2}\right)
$$


The CFFs are convolutions of the respective twist-2 GPDs $H, \widetilde{H}$ or $E$, with perturbatively calculable hard-scattering amplitudes. These amplitudes have been calculated to next-toleading order in $\alpha_{\mathrm{s}}[13], 14$, 15]. The contributions from the CFFs $\widetilde{\mathcal{H}}$ and $\mathcal{E}$ to the amplitude $M^{1,1}$ are kinematically suppressed compared to that from the CFF $\mathcal{H}$ at small values of $x_{\mathrm{B}}$ and $t$, respectively.

In addition to $s_{1}^{\mathrm{I}}$ and $c_{1}^{\mathrm{I}}$, the only other Fourier coefficients related to quark-helicityconserving twist-2 GPDs are $c_{0}^{\mathrm{I}}$ and $c_{0}^{\mathrm{DVCS}}$. The coefficient $c_{0}^{\mathrm{I}}$ is also related to $M^{1,1}$. Considering only the dominant $\mathrm{CFF} \mathcal{H}, c_{0}^{\mathrm{I}}$ is directly proportional to $c_{1}^{\mathrm{I}}$ via the factor $k$ defined above:

$$
c_{0}^{\mathrm{I}} \propto-k c_{1}^{\mathrm{I}} .
$$

The coefficients $s_{2}^{\mathrm{I}}, c_{2}^{\mathrm{I}}, s_{1}^{\mathrm{DVCS}}$ and $c_{1}^{\mathrm{DVCS}}$ are related to twist-3 GPDs. The coefficient $s_{2}^{\mathrm{I}}$ $\left(c_{2}^{\mathrm{I}}\right)$ is proportional to the imaginary (real) part of the helicity non-conserving amplitudes $M^{0, \pm 1}$, corresponding to the virtual photon being longitudinal. Conservation of angular momentum is ensured by either the exchange of an additional gluon (genuine or dynamic twist-3) or by the fact that quarks can carry non-zero orbital angular momentum along the collision axis (kinematically suppressed by the same order in $1 / Q$ ), which is possible due to the transverse momentum involved. The part of the twist-3 GPD associated with the latter picture can be related to the twist-2 quark GPDs using the Wandzura-Wilczek (WW) approximation [16] and thus is also known as the WW part of the twist-3 contribution [17].

The Fourier coefficient $c_{3}^{\mathrm{I}}$ is proportional to the real part of the amplitudes $M^{1,-1}$ and $M^{-1,1}$, which do not conserve photon helicity, i.e., both photons are transverse and they have opposite helicity. The induced two units of angular momentum can be accommodated by gluon helicity-flip. Gluon helicity-flip GPDs do not mix with quark GPDs via $Q^{2}$ evolution and thus probe the gluonic properties of the nucleon [10. They appear at leading twist, but are suppressed by a factor $\alpha_{s} / \pi$. In addition, as in the case of the coefficients discussed above that are kinematically suppressed by $1 / Q$, it is possible that the two participating quarks complete the conservation of angular momentum if they carry orbital angular momentum. As they have to account for two units of angular momentum instead of one as above, this process appears at twist-4. The associated twist-4 GPD was found to be calculable in terms of twist-2 quark GPDs using the WW approximation [18]. Similarly, the Fourier coefficient $c_{2}^{\text {DVCS }}$ arises from the twist-2 gluon helicity-flip GPDs with possible contributions from twist-4 quark GPDs.

\section{Asymmetries}

Previous measurements with a longitudinally (L) polarized positron \{electron \} beam by HERMES [19] \{CLAS [20, 21, 22] $\}$ on an unpolarized (U) proton target provided access to a combination of $s_{1}^{\mathrm{I}}$ and $s_{1}^{\mathrm{DVCS}}$ via the single-charge beam-helicity asymmetry, also denoted 
as the Beam-Spin Asymmetry (BSA):

$$
\begin{aligned}
& \mathcal{A}_{\mathrm{LU}}\left(\phi, e_{\ell}\right) \equiv \frac{d \sigma^{\rightarrow}-d \sigma^{\leftarrow}}{d \sigma^{\rightarrow}+d \sigma^{\leftarrow}} \\
& =\frac{-e_{\ell} \frac{K_{\mathrm{I}}}{\mathcal{P}_{1}(\phi) \mathcal{P}_{2}(\phi)}\left[\sum_{n=1}^{2} s_{n}^{\mathrm{I}} \sin (n \phi)\right]+\frac{1}{Q^{2}} s_{1}^{\mathrm{DVCS}} \sin \phi}{\frac{1}{\mathcal{P}_{1}(\phi) \mathcal{P}_{2}(\phi)}\left[K_{\mathrm{BH}} \sum_{n=0}^{2} c_{n}^{\mathrm{BH}} \cos (n \phi)-e_{\ell} K_{\mathrm{I}} \sum_{n=0}^{3} c_{n}^{\mathrm{I}} \cos (n \phi)\right]+\frac{1}{Q^{2}} \sum_{n=0}^{2} c_{n}^{\mathrm{DVCS}} \cos (n \phi)} .
\end{aligned}
$$

Here, $\sigma^{\rightarrow}\left(\sigma^{\leftarrow}\right)$ denotes the cross section for a beam with positive (negative) helicity. Predominant $\sin \phi$ dependences with opposite sign have been observed at the two experiments, indicating the dominance of the interference term involving $e_{\ell} \cdot s_{1}^{\mathrm{I}}$. However, quantitative access to $s_{1}^{\mathrm{I}}$ is complicated by the presence of $s_{1}^{\text {DVCS }}$, which is a higher twist-contribution but possibly significant, and by the presence of $c_{1}^{\mathrm{I}}$ and $c_{0}^{\mathrm{I}}$, i.e., the other Fourier coefficients of interest appearing at leading twist (see Eqs. 1.6 and 1.8).

This entanglement can be avoided by defining the charge-difference beam-helicity asymmetry [23]:

$$
\begin{aligned}
\mathcal{A}_{\mathrm{LU}}^{\mathrm{I}}(\phi) \equiv & \frac{\left(d \sigma^{+\rightarrow}-d \sigma^{+\leftarrow}\right)-\left(d \sigma^{-\rightarrow}-d \sigma^{-\leftarrow}\right)}{\left(d \sigma^{+\rightarrow}+d \sigma^{+\leftarrow}\right)+\left(d \sigma^{-\rightarrow}+d \sigma^{-\leftarrow}\right)} \\
= & -\frac{K_{\mathrm{I}}}{\mathcal{P}_{1}(\phi) \mathcal{P}_{2}(\phi)}\left[\sum_{n=1}^{2} s_{n}^{\mathrm{I}} \sin (n \phi)\right] \\
\frac{K_{\mathrm{BH}}}{\mathcal{P}_{1}(\phi) \mathcal{P}_{2}(\phi)} \sum_{n=0}^{2} c_{n}^{\mathrm{BH}} \cos (n \phi)+\frac{1}{Q^{2}} \sum_{n=0}^{2} c_{n}^{\mathrm{DVCS}} \cos (n \phi) &
\end{aligned}
$$

where the additional $+(-)$ superscript on the cross-sections denotes the charge of the lepton beam. This asymmetry has the important advantages that the $\sin \phi$ dependence in the numerator stems solely from the interference term, as the (higher-twist) $\sin \phi$ dependence of the squared DVCS term cancels, and the denominator no longer contains the leading terms $c_{1}^{\mathrm{I}}$ and $c_{0}^{\mathrm{I}}$. Therefore it gives direct access to linear combinations of GPDs, while another charge-averaged asymmetry related to the squared DVCS term provides access to bilinear combinations of GPDs:

$$
\begin{aligned}
\mathcal{A}_{\mathrm{LU}}^{\mathrm{DVCS}}(\phi) \equiv & \frac{\left(d \sigma^{+\rightarrow}-d \sigma^{+\leftarrow}\right)+\left(d \sigma^{-\rightarrow}-d \sigma^{-\leftarrow}\right)}{\left(d \sigma^{+\rightarrow}+d \sigma^{+\leftarrow}\right)+\left(d \sigma^{-\rightarrow}+d \sigma^{-\leftarrow}\right)} \\
= & \frac{\frac{1}{Q^{2}} s_{1}^{\mathrm{DVCS}} \sin \phi}{\frac{K_{\mathrm{BH}}}{\mathcal{P}_{1}(\phi) \mathcal{P}_{2}(\phi)} \sum_{n=0}^{2} c_{n}^{\mathrm{BH}} \cos (n \phi)+\frac{1}{Q^{2}} \sum_{n=0}^{2} c_{n}^{\mathrm{DVCS}} \cos (n \phi)} .
\end{aligned}
$$

The previously extracted [24, 25] Beam-Charge Asymmetry (BCA)

$$
\mathcal{A}_{\mathrm{C}}(\phi) \equiv \frac{d \sigma^{+}-d \sigma^{-}}{d \sigma^{+}+d \sigma^{-}}=\frac{-\frac{K_{\mathrm{I}}}{\mathcal{P}_{1}(\phi) \mathcal{P}_{2}(\phi)} \sum_{n=0}^{3} c_{n}^{I} \cos (n \phi)}{\frac{K_{\mathrm{BH}}}{\mathcal{P}_{1}(\phi) \mathcal{P}_{2}(\phi)} \sum_{n=0}^{2} c_{n}^{\mathrm{BH}} \cos (n \phi)+\frac{1}{Q^{2}} \sum_{n=0}^{2} c_{n}^{\mathrm{DVCS}} \cos (n \phi)}
$$


provides access to the real part of the DVCS amplitude via $c_{n}^{\mathrm{I}}$.

\section{Event selection}

The data were collected during the years 1996-2005 with the HERMES spectrometer 26] using the longitudinally polarized $27.6 \mathrm{GeV}$ electron and positron beams provided by the HERA accelerator facility at DESY. The hydrogen gas target was either unpolarized, longitudinally or transversely nuclear-polarized. However, the time averaged polarization of the polarized targets was negligible, while the rapid $(60-180 \mathrm{~s})$ reversal of the polarization direction minimized polarization bias due to detector effects. The polarization direction of the beam was reversed about every two months. The integrated luminosity for the electron (positron) data sample corresponds to about $106 \mathrm{pb}^{-1}\left(292 \mathrm{pb}^{-1}\right)$ with an average magnitude of the beam polarization of $30.0 \%$ (49.4\%). The latter has a mean fractional systematic uncertainty of $2.8 \%$.

A brief description of the event selection is given here. More details can be found in Refs. [24, 27]. Events are selected with exactly one photon producing an energy deposition larger than $5 \mathrm{GeV}(1 \mathrm{MeV})$ in the calorimeter (preshower detector) and one charged track, identified as the scattered lepton, in the kinematic range $1 \mathrm{GeV}^{2}<Q^{2}<10 \mathrm{GeV}^{2}, 0.03<$ $x_{B}<0.35, W>3 \mathrm{GeV}$ and $\nu<22 \mathrm{GeV}$. Here, $W$ denotes the invariant mass of the initial photon-nucleon system and $\nu$ denotes the virtual-photon energy in the target rest frame. The angle $\theta_{\gamma^{*} \gamma}$ between the real and the virtual photon is constrained to be between 5 and $45 \mathrm{mrad}$. The recoiling proton is not detected. An 'exclusive' sample of events is selected by the requirement that the squared missing mass $M_{X}^{2}$ of the reaction $e^{ \pm} p \rightarrow e^{ \pm} \gamma X$ corresponds to the squared proton mass. The resolution in $M_{X}^{2}$ is limited by the energy resolution of the real photon in the calorimeter. Correspondingly, the exclusive region is defined as $-(1.5 \mathrm{GeV})^{2}<M_{X}^{2}<(1.7 \mathrm{GeV})^{2}$, as determined from signal-to-background studies using a Monte Carlo (MC) simulation. For elastic events (leaving the proton intact), the kinematic relationship between the energy and direction of the real photon permits $t$ to be calculated without using the measured energy of the real photon, which is the quantity subject to the largest uncertainty. Thus, the value of $t$ is calculated as

$$
t=\frac{-Q^{2}-2 \nu\left(\nu-\sqrt{\nu^{2}+Q^{2}} \cos \theta_{\gamma^{*} \gamma}\right)}{1+\frac{1}{M_{p}}\left(\nu-\sqrt{\nu^{2}+Q^{2}} \cos \theta_{\gamma^{*} \gamma}\right)}
$$

for the exclusive event sample. The quantity $-t$ is required to be smaller than $0.7 \mathrm{GeV}^{2}$. The error caused by applying this expression to inelastic events is accounted for in the MC simulation that is used to calculate the fractional contribution of background processes per kinematic bin in $x_{\mathrm{B}}, Q^{2}$, and $-t$.

\section{Extraction of asymmetry amplitudes}

The experimental yield $\mathcal{N}$ can be parameterized as

$$
\mathcal{N}\left(e_{\ell}, P_{\ell}, \phi\right)=\mathcal{L}\left(e_{\ell}, P_{\ell}\right) \eta\left(e_{\ell}, \phi\right) \sigma_{\mathrm{UU}}(\phi) \times\left[1+P_{\ell} \mathcal{A}_{\mathrm{LU}}^{\mathrm{DVCS}}(\phi)+e_{\ell} P_{\ell} \mathcal{A}_{\mathrm{LU}}^{\mathrm{I}}(\phi)+e_{\ell} \mathcal{A}_{\mathrm{C}}(\phi)\right] .
$$


Here, $\mathcal{L}$ is the integrated luminosity, $\eta$ the detection efficiency, $P_{\ell}$ the beam polarization and $\sigma_{\mathrm{UU}}$ the cross section for an unpolarized target averaged over both beam charges and helicities. The asymmetries $\mathcal{A}_{\mathrm{LU}}^{\mathrm{I}}(\phi), \mathcal{A}_{\mathrm{LU}}^{\mathrm{DVCS}}(\phi)$, and $\mathcal{A}_{\mathrm{C}}(\phi)$ relate to the Fourier coefficients in Eqs. 1.2 1.4 according to Eqs. 2.2 2.4. They are expanded in $\phi$ as

$$
\begin{gathered}
\mathcal{A}_{\mathrm{LU}}^{\mathrm{I}}(\phi) \simeq \sum_{n=1}^{2} A_{\mathrm{LU}, \mathrm{I}}^{\sin (n \phi)} \sin (n \phi)+\sum_{n=0}^{1} A_{\mathrm{LU}, \mathrm{I}}^{\cos (n \phi)} \cos (n \phi), \\
\mathcal{A}_{\mathrm{LU}}^{\mathrm{DVCS}}(\phi) \simeq \sum_{n=1}^{2} A_{\mathrm{LU}, \mathrm{DVCS}}^{\sin (n \phi)} \sin (n \phi)+\sum_{n=0}^{1} A_{\mathrm{LU}, \mathrm{DVCS}}^{\cos (n \phi)} \cos (n \phi), \\
\mathcal{A}_{\mathrm{C}}(\phi) \simeq \sum_{n=0}^{3} A_{\mathrm{C}}^{\cos (n \phi)} \cos (n \phi) \quad+A_{\mathrm{C}}^{\sin \phi} \sin \phi,
\end{gathered}
$$

where the approximation is due to the truncation of the in general infinite Fourier series caused by the azimuthal dependences in the denominators of Eqs. 2.2 2.4. The asymme-

try amplitudes $A_{\mathrm{LU}, \mathrm{I}}^{\sin \phi}, A_{\mathrm{C}}^{\cos \phi}$ and $A_{\mathrm{C}}^{\cos (0 \phi)}$ relate to the twist-2 Fourier coefficients of the interference term appearing in Eq. 1.4 and further developed in Eqs. 1.5, 1.6 and 1.8, respectively. The asymmetry amplitudes $A_{\mathrm{LU}, \mathrm{DVCS}}^{\sin \phi}, A_{\mathrm{LU}, \mathrm{I}}^{\sin (2 \phi)}, A_{\mathrm{C}}^{\cos (2 \phi)}$ and $A_{\mathrm{C}}^{\cos (3 \phi)}$ are related to other Fourier coefficients in Eqs. 1.3 and 1.4, which are also explained above. The remaining asymmetry amplitudes are expected to be zero but were introduced to test for instrumentally induced harmonics. (The asymmetry amplitude $A_{\mathrm{LU}, \mathrm{DVCS}}^{\sin (2 \phi)}$ can, in addition, arise through the interplay of numerator and denominator in Eq. 2.3 if the twist-3 Fourier coefficient $s_{1}^{\text {DVCS }}$ has a sizeable value.) Comparison of predictions based on GPD models to data for either asymmetry amplitudes or the Fourier coefficients in Eqs. 1.3 and 1.4 provides similar information, as the asymmetry amplitudes relate to the corresponding Fourier coefficients in an approximately model-independent way. This is due to the fact that the $\mathrm{BH}$ coefficients $c_{n}^{\mathrm{BH}}$ and lepton propagators are precisely calculable, and that in the kinematic region of HERMES the contributions from the squared DVCS term in the denominators of Eqs. 2.2 2.4 are expected to be much smaller than the BH contributions [28].

The extraction of the asymmetry amplitudes in each kinematic bin of $x_{B}, Q^{2}$ and $t$ is based on the maximum likelihood technique [29], providing a bin-free fit in $\phi$ to the data. Its application here is explained in detail in Ref. [25]. In the fit, weights were introduced to account for luminosity imbalances with respect to beam charge and polarization. No balancing procedure was required for the target polarization, as the time averaged polarization was negligible as mentioned above.

\section{Background corrections and systematic uncertainties}

Each extracted asymmetry amplitude $A$ is corrected, in each kinematic bin, for the contribution of semi-inclusive and exclusive background, which is mostly due to the production of $\pi^{0}$ and $\eta$ mesons. The background-corrected amplitude is calculated as

$$
A_{\text {corr }}=\frac{A-f_{\mathrm{semi}} A_{\mathrm{semi}}-f_{\mathrm{excl}} A_{\mathrm{excl}}}{1-f_{\mathrm{semi}}-f_{\mathrm{excl}}} .
$$


The fraction $f_{\text {semi }}$ of semi-inclusive background per bin is calculated using a MC simulation described in detail in Ref [25]. It varies between $0.6 \%$ and $12.7 \%$ depending on the kinematic bin, with an average value of 3.3\%. Based on the model in Ref. [30], the fraction $f_{\text {excl }}$ of exclusive background is estimated in each kinematic bin and found to be below $0.7 \%$ everywhere. A direct search for exclusive neutral pions in the HERMES data supports this estimate [31]. The semi-inclusive (exclusive) background can have a non-zero asymmetry $A_{\text {semi }}\left(A_{\text {excl }}\right)$, as has been measured for, e.g., the semi-inclusive production of $\pi^{0}$ mesons, which exhibits a sinusoidal $\phi$ dependence on the beam helicity 32]. The beam-charge-dependent background asymmetry is zero at leading order QED. Hence the contributions from semi-inclusive and exclusive background constitute a dilution of $\mathcal{A}_{\mathrm{C}}$ and effectively also of $\mathcal{A}_{\mathrm{LU}}^{\mathrm{I}}$, as the latter involves only charge differences. In order to correct $\mathcal{A}_{\mathrm{LU}}^{\mathrm{DVCS}}$ for the semi-inclusive background, the size of the beam-helicity asymmetry for this background is extracted from data by reconstructing neutral pions with a large fractional energy $E_{\pi^{0}} / \nu>0.8$ (see Ref. [25] for details). For the exclusive background, the asymmetry cannot be extracted from data due to the small yield of exclusive pions. As the asymmetry is in the range $[-1,1]$, a value of zero is assumed with a "statistical" uncertainty of $2 / \sqrt{12}$, i.e., one standard deviation for a uniform distribution. The statistical uncertainty on the total correction due to the statistical uncertainties in the background fractions and asymmetries is propagated as a contribution to the final statistical uncertainty. In addition, half the size of the actual correction is assigned as systematic uncertainty to account for assumptions and approximations inherent in the approach.

The dominant systematic uncertainty for the amplitude $A_{\mathrm{LU}, \mathrm{DVCS}}^{\sin \phi}$ is due to the background correction. In the case of $A_{\mathrm{LU}, \mathrm{I}}^{\sin (n \phi)}$ and $A_{\mathrm{C}}^{\cos (n \phi)}$, the systematic uncertainty is predominantly due to the combined contributions of possible deviations of the detector and/or the beam from their nominal positions ('alignment'), detector acceptance including smearing, and finite bin width in $x_{\mathrm{B}}, t$ and $Q^{2}$. The systematic uncertainty arising from these combined contributions is estimated by MC simulations using the GPD model described in Ref [33]. Note that a mistake has been found in this GPD model [34]. However, the model of Ref. [33] described HERMES beam-charge [25] and preliminary (single charge) beam-helicity asymmetries [35] and thus is considered adequate for systematic studies. In each bin, the systematic uncertainty is taken as the difference between the model predictions at the mean kinematic value of that bin and the respective amplitude extracted from the reconstructed $\mathrm{MC}$ data.

Further systematic uncertainties arise from an observed relative shift of the squared missing-mass spectra between the electron and positron sample, with a magnitude of approximately $0.2 \mathrm{GeV}^{2}$ 36. The boundaries defining the exclusive sample in the missing mass spectra were adjusted to account for this shift. One quarter of the effect on the extracted asymmetries is applied as systematic uncertainty. The impact of both trigger and tracking inefficiencies was studied and found to be negligible. Also not included is any contribution due to additional QED vertices, as the most significant of these has been estimated to be negligible in the case of helicity asymmetries [37]. The systematic uncertainties are added in quadrature and given in Table 1 . 


\begin{tabular}{|l|r|c|c|c|}
\hline Amplitude & $A \pm \delta$ (stat.) $\pm \delta$ (syst.) & Missing mass shift & Background corr. & Alignment, acceptance, bin width \\
\hline \hline$A_{\mathrm{LU}, \mathrm{I}}^{\sin \phi}$ & $-0.224 \pm 0.028 \pm 0.020$ & 0.001 & 0.005 & 0.019 \\
\hline$A_{\mathrm{LU}, \mathrm{DVCS}}^{\sin }$ & $0.043 \pm 0.028 \pm 0.004$ & 0.000 & 0.004 & 0.000 \\
\hline$A_{\mathrm{C}}^{\cos (0 \phi)}$ & $-0.020 \pm 0.006 \pm 0.008$ & 0.004 & 0.000 & 0.007 \\
\hline$A_{\mathrm{C}}^{\cos \phi}$ & $0.055 \pm 0.009 \pm 0.004$ & 0.001 & 0.001 & 0.003 \\
\hline
\end{tabular}

Table 1: Overall values of the asymmetry amplitudes of greatest interest, at average kinematics $\langle-t\rangle=0.12 \mathrm{GeV}^{2},\left\langle x_{B}\right\rangle=0.09$, and $\left\langle Q^{2}\right\rangle=2.37 \mathrm{GeV}^{2}$. The rightmost three columns present dominant contributions to the systematic uncertainties. Not included is a $2.8 \%$ scale uncertainty of the beam-helicity asymmetries due to the beam polarization measurement.

\section{Results}

Table 1 presents the asymmetry amplitudes of greatest interest extracted in the entire HERMES kinematic acceptance ("overall" results). The $\sin \phi$ amplitude of the beamhelicity asymmetry sensitive to the interference term is shown in the first row of Fig. 2 . It exhibits a large overall value of $A_{\mathrm{LU}, \mathrm{I}}^{\mathrm{sin} \phi}=-0.224 \pm 0.028$ (stat.) \pm 0.020 (sys.), with no significant dependence on any of the kinematic variables $-t, x_{\mathrm{B}}$, and $Q^{2}$. This implies a rather strong dependence of this amplitude on $t$ for smaller values of $-t$, as the asymmetry amplitude has to vanish in the limit of vanishing $-t$ due to the vanishing factor $k$ in Eq. 1.5. (In the limit of vanishing $t, c_{0}^{\mathrm{BH}}$ remains finite and the dependences of $K_{\mathrm{BH}}, K_{\mathrm{I}}, \mathcal{P}_{1}(\phi)$ and $\mathcal{P}_{2}(\phi)$ on $t$ cancel in Eq. 2.2.) The $\sin \phi$ amplitude of the beam-helicity asymmetry sensitive to the squared DVCS term is shown in the second row of Fig. 2. It also shows no kinematic dependence, with an overall value of $A_{\mathrm{LU} \text {,DVCS }}^{\sin \phi}=0.043 \pm 0.028$ (stat.) \pm 0.004 (sys.). As explained above (see Eq. 2.1), the beam-helicity asymmetries measured previously with a single beam-charge are sensitive only to the combination of the results presented here, i.e., the single-beam-charge results are given as

$$
A_{\mathrm{LU}}^{\sin \phi}\left(e_{\ell}\right) \approx e_{l} A_{\mathrm{LU}, \mathrm{I}}^{\sin \phi}+A_{\mathrm{LU}, \mathrm{DVCS}}^{\sin \phi}
$$

if the contributions $c_{n}^{\mathrm{I}}$ from the interference term in the denominator of Eq. 2.1 can be neglected. Previous HERMES measurements [24, 25] found these contributions to be small compared to the remainder of the denominator, and a more precise constraint is presented below. Using the present data, the separate analysis of the positron \{electron $\}$ data yields values for $A_{\mathrm{LU}}^{\sin \phi}\left(e_{\ell}\right)$ of $-0.177 \pm 0.022$ (stat.) $\{0.255 \pm 0.051$ (stat.) $\}$, in agreement with $-0.181 \pm 0.046$ (stat.) $\{0.267 \pm 0.065$ (stat.) $\}$ calculated from Eq. 6.1 neglecting correlations from the commonality of the data sets.

The $\sin 2 \phi$ amplitude of $A_{\mathrm{LU}, \mathrm{I}}$ is shown in the third row of Fig. 2. It has an overall value consistent with zero $(-0.035 \pm 0.028 \pm 0.002)$ and thus, as expected, is much smaller than the corresponding $\sin \phi$ amplitude. Those asymmetry amplitudes included in Eqs. 4.24 .4 as tests for instrumental effects are found to be compatible with zero.

The data in Fig. 2 are compared with theoretical calculations to leading order in QED and QCD. In the GPD based model (VGG) described in Refs. 30, 38], the dependences on $\xi$ and $t$ are factorized while those on $x$ and $t$ may be entangled when a Regge-motivated ansatz is invoked. The model is formulated as a double distribution [1, 3] complemented by 


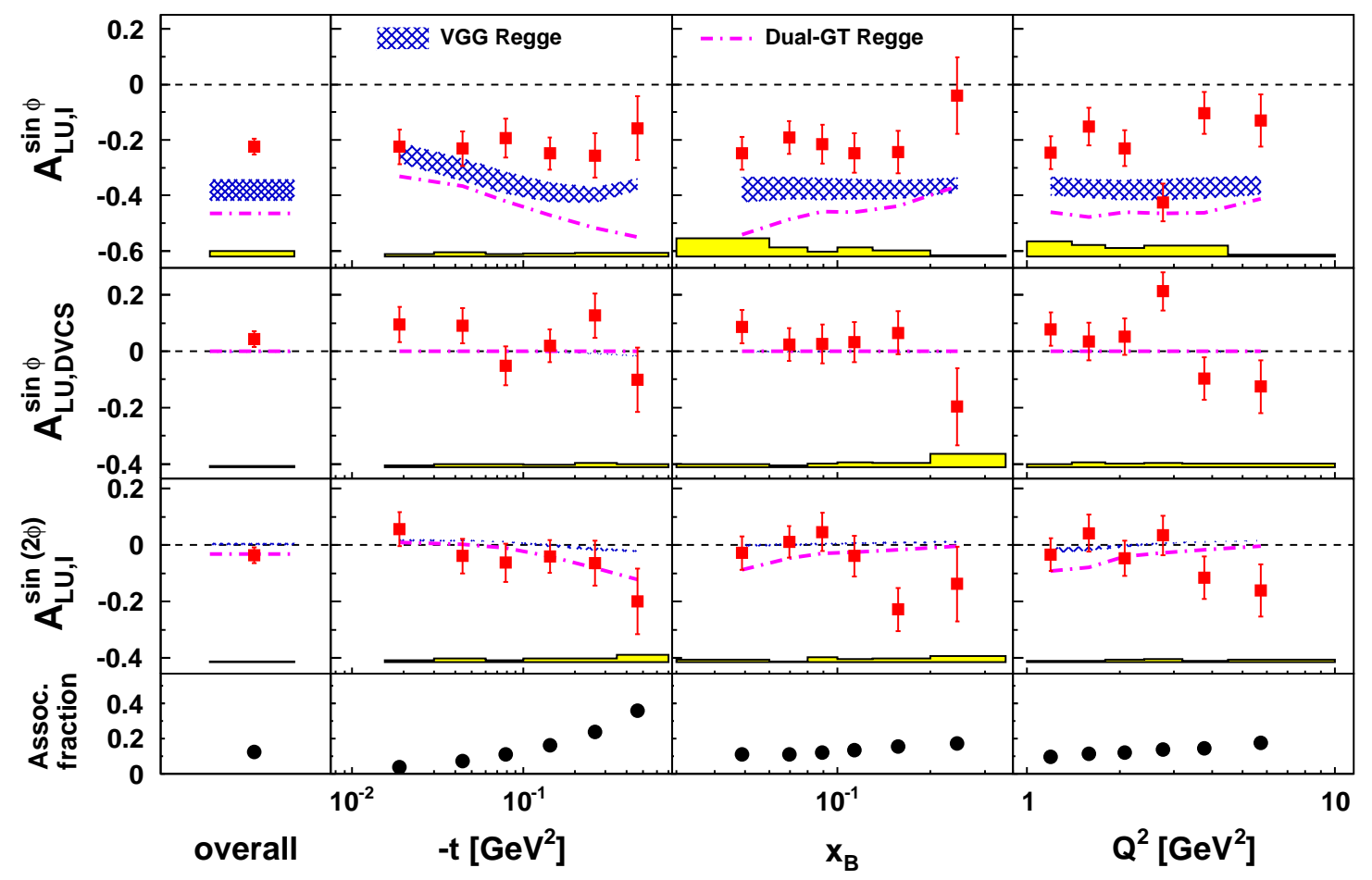

Figure 2: The first (second) row shows the $\sin \phi$ amplitude of the beam-helicity asymmetry $A_{\mathrm{LU}, \mathrm{I}}$ ( $\left.A_{\mathrm{LU}, \mathrm{DVCS}}\right)$, which is sensitive to the interference term (squared DVCS term), extracted from the 1996-2005 hydrogen data in the entire experimental acceptance, and as a function of $-t, x_{\mathrm{B}}$, and $Q^{2}$. The third row shows the $\sin 2 \phi$ amplitude of $A_{\mathrm{LU}, \mathrm{I}}$. The error bars (bands) represent the statistical (systematic) uncertainties. Not included is a $2.8 \%$ scale uncertainty due to the beam polarization measurement. The calculations are based on the recently corrected minimal implementation [33, 34] of a dual-parameterization GPD model (Dual-GT) and on a GPD model [30, 38 based on doubledistributions (VGG). Both models use a Regge-motivated $t$-dependence. The band for the VGG model results from varying the parameters $b_{\text {val }}$ and $b_{\text {sea }}$ between unity and infinity. The bottom row shows the fractional contribution of associated BH production as obtained from a MC simulation.

a D-term [39], where the kernel of the double distribution contains a profile function 40, 41] that determines the dependence on $\xi$, controlled by a parameter $b$ 42]. In the limit $b \rightarrow \infty$ the GPD is independent of $\xi$. Note that $b_{\text {val }}\left(b_{\text {sea }}\right)$ is a free parameter for the valence (sea) quarks and thus can be used as a fit parameter in the extraction of GPDs from hard-electroproduction data.

In each kinematic bin, a range of theoretical predictions was calculated 43. by varying the model parameters of only the GPD $H$, since these data are sensitive mostly to this GPD as explained above. Variants of the model are distinguished by differences in the $t$ dependence of the GPD $H$, for which either a simple ansatz is used where the $t$ dependence factorizes from the dependence on the other kinematic variables, or the Regge-motivated ansatz is employed. Since the differences are found to be small for all amplitudes shown in Fig. 2, only the results based on the latter ansatz (VGG Regge) are displayed. The 
broad width of the bands is due to the fact that the parameters $b_{\text {val }}$ and $b_{\text {sea }}$ were varied between unity and infinity, with the variation in $b_{\text {sea }}$ having the strongest effect. Note that including or neglecting the D-term in the GPD model does not change the result since it contributes only to the real part of the DVCS amplitude. The other model presented here (Dual-GT) is based on the corrected [34] minimal implementation [33] of the dual parameterization GPD model [44], in which the dependence on $\xi$ is factorized from the dependences on $x$ and $t$. The $t$ dependence in this model is also Regge-motivated. All models overestimate the magnitude of $A_{\mathrm{LU}, \mathrm{I}}^{\sin \phi}$ by approximately a factor of two. They are consistent with the observed shapes of the kinematic dependences on $x_{B}$ and $Q^{2}$ (but not $t$ ). The part of the VGG band closest to the data, i.e., with the smallest absolute amplitude, corresponds to $b_{\text {sea }}=\infty$. Note that these models are for the elastic part of the cross section only while this measurement includes associated production in which the nucleon in the final state is excited to a resonant state. In the following it is considered whether the contribution from associated production can account for the observed discrepancies between model predictions and data.

The bottom row of Fig. 2 shows the estimated fractional contribution from associated $\mathrm{BH}$ production in each kinematic bin, calculated using $\mathrm{MC}$ simulations described in Ref. [25]. The overall value is about $12 \%$. In an attempt to estimate $A_{\mathrm{LU}, \mathrm{I}}^{\sin \phi}$ in elastic and associated production separately, the strong dependence of the fractional contributions of elastic and associated production on the missing mass value in the exclusive region $-(1.5 \mathrm{GeV})^{2}<M_{X}^{2}<(1.7 \mathrm{GeV})^{2}$ can be utilized. The exclusive region can be split in several bins, each bin with its background-corrected amplitude $A_{\text {corr }}=f_{\text {elas }} A_{\text {elas }}+f_{\text {asso }} A_{\text {asso }}$. The fraction $f_{\text {elas }}\left(f_{\text {asso }}\right)$ of elastic (associated) production per bin is taken from the MC simulation, in which the DVCS process is not implemented because the BH cross section is expected to dominate that of DVCS not only for elastic but also for associated production 455. Assuming that the values of $A_{\mathrm{LU}, \mathrm{I}}^{\sin \phi}$ for elastic and associated production do not depend on $M_{X}^{2}$, the two unknown amplitudes $A_{\text {elas }}$ and $A_{\text {asso }}$ are extracted from the five equations corresponding to the five $M_{X}^{2}$-bins. The resulting overall $\sin \phi$ amplitude from elastic production is found to be $-0.209 \pm 0.066$ and thus hardly differs from that reported in Table 1, while the one from associated production can only be constrained to be between -0.68 and 0.09 within one standard deviation in the statistical uncertainty. According to theoretical calculations [45] a correction factor of 1.1 has to be applied to the measured beam-helicity asymmetry in HERMES kinematics due to the $\Delta$ resonance region $(W<1.35 \mathrm{GeV})$. For the associated DVCS amplitude these calculations are based on a model for transition GPDs, which are related to those on the nucleon within this model. Thus neither the extracted $\sin \phi$ amplitudes $A_{\text {elas }}$ and $A_{\text {asso }}$ nor model calculations support extreme scenarios in which the $\sin \phi$ amplitude from associated production has an overall value of unity, which would be required to obtain a $\sin \phi$ amplitude for elastic production as large as the value -0.39 or more predicted by the models shown. Thus associated production cannot account for the overestimate of the asymmetry amplitudes by the models.

A promising alternative to comparing the data with existing models is to use a flexible GPD parameterization and perform a global fit to all DVCS data. First steps in this 
direction have been made [46, 47, 48, 49, 50], one of which found that a preliminary HERMES result on the $\sin \phi$ amplitude of the beam helicity asymmetry for a single charge (positron) [35] can be described by a fit to other DVCS data 48]. In order to provide additional input for future fits, in particular for the entangled $\xi$ and $-t$ dependences of GPDs, the amplitudes already presented in Fig. 2 are shown in Fig. 3 as a function of $-t$

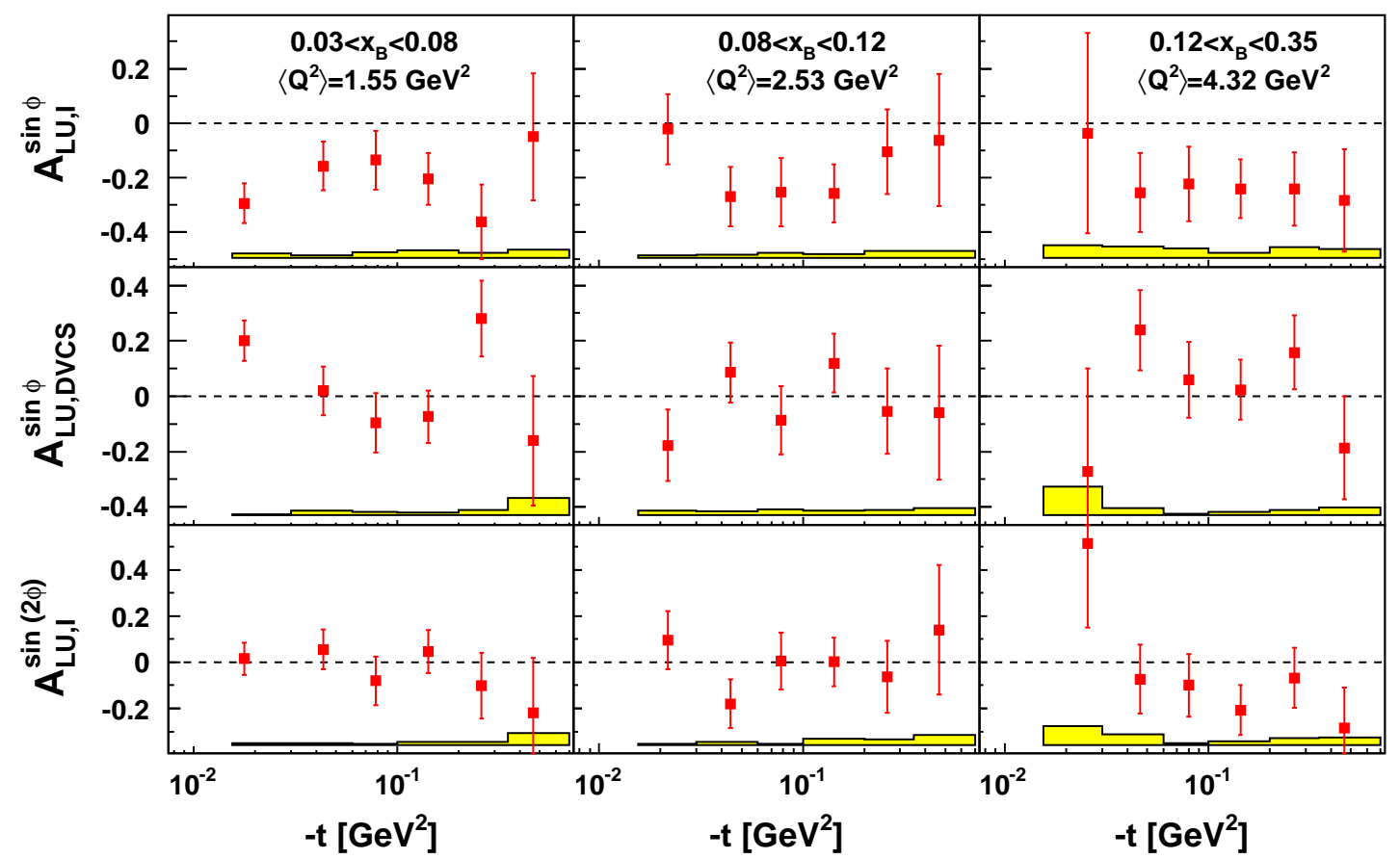

Figure 3: The first (second) row shows the $\sin \phi$ amplitude of the beam-helicity asymmetry $A_{\mathrm{LU}, \mathrm{I}}$ ( $\left.A_{\mathrm{LU}, \mathrm{DVCS}}\right)$ sensitive to the interference term (squared DVCS term), extracted from the 19962005 hydrogen data as a function of $-t$ for three $x_{\mathrm{B}}$ ranges. Correspondingly, the third row shows the $\sin (2 \phi)$ amplitude of $A_{\mathrm{LU}, \mathrm{I}}$. The error bars (bands) represent the statistical (systematic) uncertainties. Not included is a $2.8 \%$ scale uncertainty due to the beam polarization measurement.

for three different ranges of $x_{\mathrm{B}}$. The possibly negative $\sin 2 \phi$ amplitude for the largest $x_{\mathrm{B}}$ bins in Fig. 2 is found to be independent of $t$ in the lower right panel of Fig. 3 .

The $\cos (n \phi)$ amplitudes $(n=0-3)$ of the beam charge asymmetry are shown in Fig. 4 . The $\cos (0 \phi)$ and the $\cos \phi$ amplitudes, i.e., the amplitudes related to twist-2 GPDs, are zero at small values of $-t$ and become non-zero with increasing values of $-t$, with opposite sign and smaller magnitude for $\cos (0 \phi)$ as expected from Eq. 1.8. It is interesting to note that $A_{\mathrm{C}}^{\cos \phi}$ and $A_{\mathrm{LU}, \mathrm{I}}^{\sin \phi}$ show a fundamentally different dependence on $-t$, despite relating to the real and imaginary parts of the twist-2 helicity-conserving DVCS amplitude via the same factor $k \propto \sqrt{-t} / Q$ in Eq. 1.6 and Eq. 1.5, respectively. The $\cos \phi$ amplitude does not exhibit any kinematic dependence on $x_{\mathrm{B}}$ or $Q^{2}$. It is in agreement with the earlier HERMES measurements based on subsamples of the data used in this analysis [24, 25]. The $\cos (2 \phi)$ amplitude, which is related to twist-3 GPDs, is suppressed as expected and found to be compatible with zero. Also, the $\cos (3 \phi)$ amplitude, which is related to gluon 


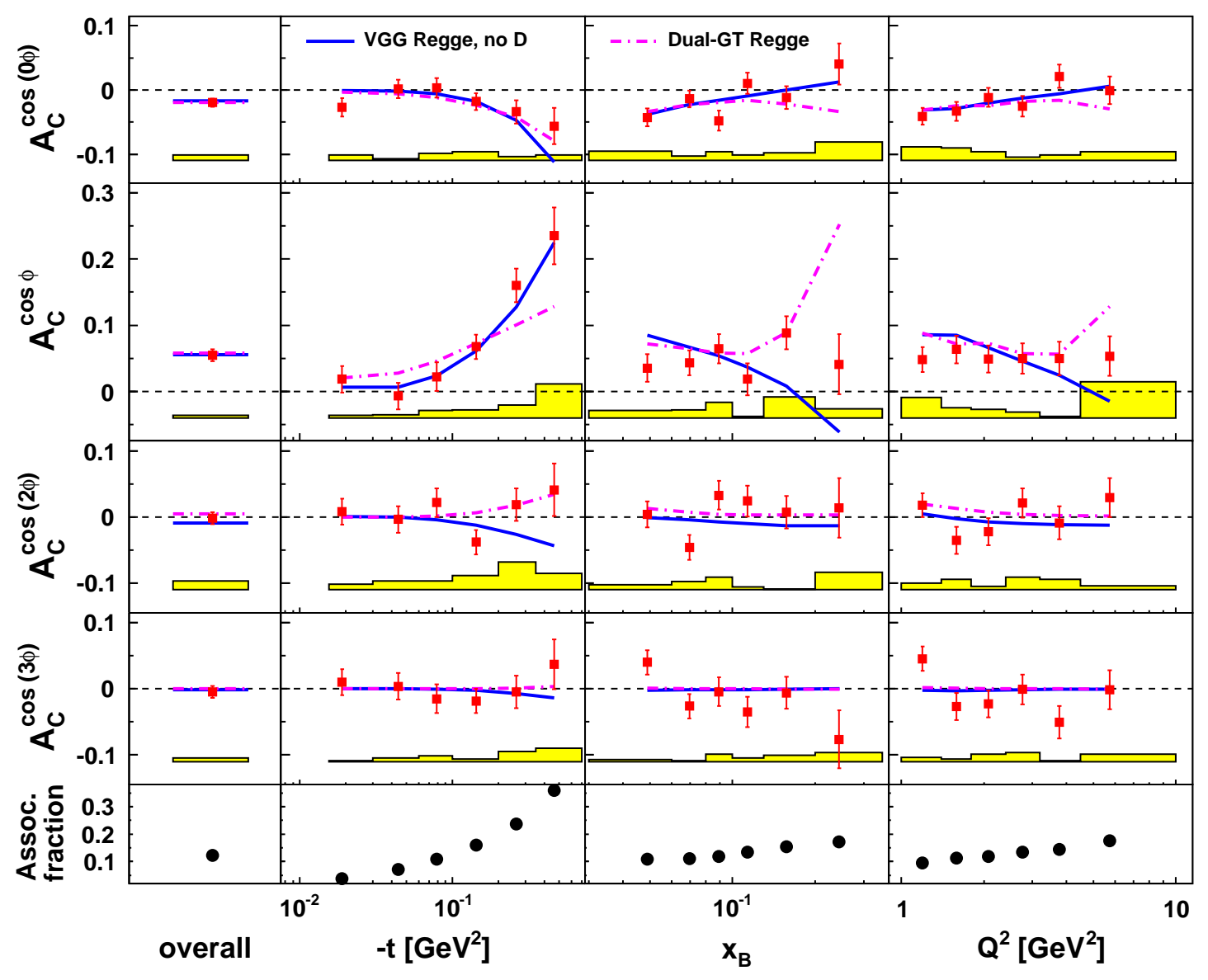

Figure 4: The $\cos (n \phi)$ amplitude $(n=0-3)$ of the beam-charge asymmetry $A_{\mathrm{C}}$, extracted from the 1996-2005 hydrogen data in the entire experimental acceptance, and as a function of $-t, x_{\mathrm{B}}$, and $Q^{2}$. The error bars (bands) represent the statistical (systematic) uncertainties. The theoretical calculations are based on the models that are unable to describe the data in Fig. 2. For the VGG model the parameter settings $b_{\text {val }}=\infty$ and $b_{\text {sea }}=1$ are used and the contribution from the D-term is set to zero. The bottom row shows the fractional contribution of associated BH production as obtained from a MC simulation.

helicity-flip GPDs, is found to be consistent with zero. No striking additional features are observed in Fig. 目 where the $\cos (n \phi)$ amplitudes are shown as a function of $-t$ for three distinct $x_{\mathrm{B}}$ ranges.

The theoretical calculations shown in Fig. 4 are based on either the Dual-GT or the VGG model. For the VGG model the parameter settings $b_{\text {val }}=\infty$ and $b_{\text {sea }}=1$ are used and the contribution from the D-term is set to zero, as only this set of parameters yields a good description of the BCA data [24, 25]. Note that the same set, in particular the setting $b_{\text {sea }}=1$, leads to amplitudes with the largest magnitude among those represented in the bands in the top row of Fig. 2, i.e., it clearly does not describe the data related to the imaginary part of the DVCS amplitude. It appears that additional degrees of freedom 


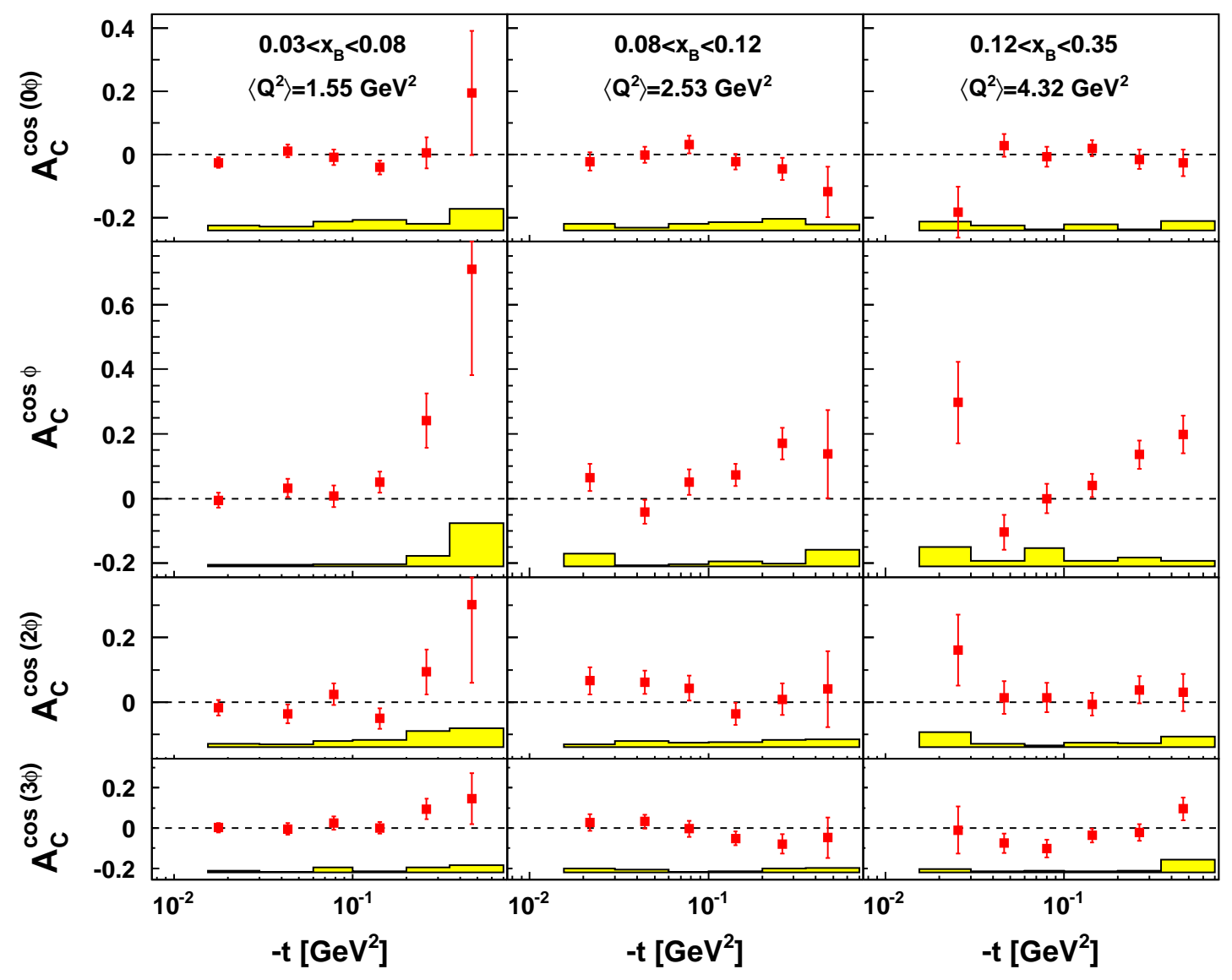

Figure 5: The $\cos (n \phi)$ amplitude $(n=0-3)$ of the beam-charge asymmetry $A_{\mathrm{C}}$, extracted from the 1996-2005 hydrogen data as a function of $-t$ for three $x_{\mathrm{B}}$ ranges. The error bars (bands) represent the statistical (systematic) uncertainties.

in the calculation of the BCA, such as the value assigned to the D-term, allow the VGG model to be tuned to resemble the BCA data. Similarly, the Dual-GT model does not describe the data in Fig. 2 but is in reasonable agreement with the BCA data shown in Fig. 4. (The sudden increase of the $\cos \phi$ amplitude predicted by this model in the highest $x_{B}$ and $Q^{2}$ bins might be due to the fact that this model is designed for small and medium values of $x_{B}$ up to 0.2.) While the increase $\{$ decrease $\}$ of the $\cos \phi\{\cos (0 \phi)\}$ amplitude with $-t$ is well reproduced within these models, the contribution of associated processes not included in these models is expected to also increase with $-t$ as shown in the bottom row.

\section{Summary}

Previously unmeasured charge-difference and charge-averaged beam-helicity asymmetries in hard electroproduction of real photons from an unpolarized proton target are extracted 
from data taken with electron and positron beams. The $\sin \phi$ amplitudes of these beamhelicity asymmetries are sensitive to the interference term (twist-2) and to the squared DVCS term (twist-3), respectively, whereas earlier measured beam-helicity asymmetries with a single beam-charge are sensitive to only their linear combination. In addition, the most precise determination of the beam-charge asymmetry is presented, which provides access to the real part of the DVCS amplitude. The GPD models presented are not able to describe the $\sin \phi$ amplitude sensitive to the interference term, while they can be adjusted to resemble the results on the beam-charge asymmetry, presumably because the model calculations have additional degrees of freedom in the latter case. The amplitudes related to higher-twist or gluon helicity-flip GPDs are found to be compatible with zero. The results presented on these charge-decomposed beam-helicity asymmetries and on the highprecision beam-charge asymmetry have the potential to considerably constrain the GPD $H$ when used in comparison with future GPD models or as input to global fits.

\section{Acknowledgments}

We gratefully acknowledge the DESY management for its support and the staff at DESY and the collaborating institutions for their significant effort. This work was supported by the FWO-Flanders and IWT, Belgium; the Natural Sciences and Engineering Research Council of Canada; the National Natural Science Foundation of China; the Alexander von Humboldt Stiftung; the German Bundesministerium für Bildung und Forschung (BMBF); the Deutsche Forschungsgemeinschaft (DFG); the Italian Istituto Nazionale di Fisica Nucleare (INFN); the MEXT, JSPS, and G-COE of Japan; the Dutch Foundation for Fundamenteel Onderzoek der Materie (FOM); the U.K. Engineering and Physical Sciences Research Council, the Science and Technology Facilities Council, and the Scottish Universities Physics Alliance; the U.S. Department of Energy (DOE) and the National Science Foundation (NSF); the Russian Academy of Science and the Russian Federal Agency for Science and Innovations; the Ministry of Economy and the Ministry of Education and Science of Armenia; and the European Community-Research Infrastructure Activity under the FP6 "Structuring the European Research Area" program (HadronPhysics, contract number RII3-CT-2004-506078). 


\section{A. Tables of Results}

\begin{tabular}{|c|c|c|c|c|c|c|c|}
\hline \multicolumn{2}{|c|}{ kinematic bin } & $\langle-t\rangle$ & $\left\langle x_{\mathrm{B}}\right\rangle$ & $\left\langle Q^{2}\right\rangle$ & $A_{\mathrm{LU}, \mathrm{I}}^{\sin \phi}$ & $A_{\mathrm{LU}, \mathrm{DVCS}}^{\sin \phi}$ & $A_{\mathrm{LU}, \mathrm{I}}^{\sin (2 \phi)}$ \\
\hline & overall & -0.12 & 0.10 & 2.46 & $-0.224 \pm 0.028 \pm 0.020$ & $0.043 \pm 0.028 \pm 0.004$ & $-0.035 \pm 0.028 \pm 0.002$ \\
\hline \multirow{6}{*}{ 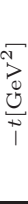 } & $0.00-0.03$ & -0.02 & 0.07 & 1.71 & $-0.225 \pm 0.062 \pm 0.010$ & $0.095 \pm 0.062 \pm 0.007$ & $0.057 \pm 0.061 \pm 0.006$ \\
\hline & $0.03-0.06$ & -0.04 & 0.09 & 2.22 & $-0.231 \pm 0.063 \pm 0.016$ & $0.091 \pm 0.062 \pm 0.010$ & $-0.039 \pm 0.061 \pm 0.012$ \\
\hline & $0.06-0.10$ & -0.08 & 0.10 & 2.44 & $-0.193 \pm 0.069 \pm 0.009$ & $-0.051 \pm 0.069 \pm 0.011$ & $-0.063 \pm 0.068 \pm 0.006$ \\
\hline & $0.10-0.20$ & -0.14 & 0.11 & 2.72 & $-0.249 \pm 0.059 \pm 0.013$ & $0.020 \pm 0.058 \pm 0.008$ & $-0.041 \pm 0.058 \pm 0.012$ \\
\hline & $0.20-0.35$ & -0.26 & 0.12 & 3.13 & $-0.256 \pm 0.080 \pm 0.013$ & $0.126 \pm 0.079 \pm 0.015$ & $-0.065 \pm 0.080 \pm 0.013$ \\
\hline & $0.35-0.70$ & -0.46 & 0.12 & 3.63 & $-0.158 \pm 0.115 \pm 0.013$ & $-0.101 \pm 0.114 \pm 0.010$ & $-0.201 \pm 0.116 \pm 0.025$ \\
\hline \multirow{6}{*}{$\underset{\forall}{\infty}$} & $0.03-0.06$ & -0.10 & 0.05 & 1.34 & $-0.248 \pm 0.060 \pm 0.067$ & $0.087 \pm 0.059 \pm 0.011$ & $-0.028 \pm 0.059 \pm 0.008$ \\
\hline & $0.06-0.08$ & -0.09 & 0.07 & 1.78 & $-0.191 \pm 0.059 \pm 0.034$ & $0.023 \pm 0.058 \pm 0.007$ & $0.011 \pm 0.057 \pm 0.003$ \\
\hline & $0.08-0.10$ & -0.11 & 0.09 & 2.30 & $-0.215 \pm 0.069 \pm 0.018$ & $0.026 \pm 0.069 \pm 0.014$ & $0.046 \pm 0.068 \pm 0.018$ \\
\hline & $0.10-0.13$ & -0.12 & 0.11 & 2.92 & $-0.248 \pm 0.071 \pm 0.032$ & $0.033 \pm 0.071 \pm 0.016$ & $-0.039 \pm 0.072 \pm 0.010$ \\
\hline & $0.13-0.20$ & -0.16 & 0.16 & 4.04 & $-0.244 \pm 0.077 \pm 0.023$ & $0.066 \pm 0.077 \pm 0.016$ & $-0.229 \pm 0.076 \pm 0.012$ \\
\hline & $0.20-0.35$ & -0.23 & 0.24 & 6.11 & $-0.040 \pm 0.139 \pm 0.005$ & $-0.196 \pm 0.137 \pm 0.048$ & $-0.138 \pm 0.132 \pm 0.022$ \\
\hline \multirow{6}{*}{ 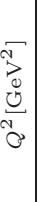 } & $1.0-1.4$ & -0.08 & 0.05 & 1.20 & $-0.247 \pm 0.059 \pm 0.055$ & $0.078 \pm 0.059 \pm 0.010$ & $-0.034 \pm 0.058 \pm 0.005$ \\
\hline & $1.4-1.8$ & -0.10 & 0.07 & 1.59 & $-0.151 \pm 0.067 \pm 0.042$ & $0.034 \pm 0.067 \pm 0.016$ & $0.042 \pm 0.066 \pm 0.004$ \\
\hline & $1.8-2.4$ & -0.11 & 0.08 & 2.08 & $-0.230 \pm 0.064 \pm 0.031$ & $0.052 \pm 0.064 \pm 0.013$ & $-0.047 \pm 0.062 \pm 0.009$ \\
\hline & $2.4-3.2$ & -0.13 & 0.10 & 2.77 & $-0.425 \pm 0.068 \pm 0.041$ & $0.212 \pm 0.068 \pm 0.015$ & $0.034 \pm 0.070 \pm 0.011$ \\
\hline & $3.2-4.5$ & -0.15 & 0.13 & 3.76 & $-0.103 \pm 0.076 \pm 0.040$ & $-0.097 \pm 0.075 \pm 0.012$ & $-0.116 \pm 0.076 \pm 0.003$ \\
\hline & $4.5-10$ & -0.22 & 0.20 & 5.75 & $-0.129 \pm 0.094 \pm 0.008$ & $-0.125 \pm 0.093 \pm 0.013$ & $-0.161 \pm 0.092 \pm 0.009$ \\
\hline
\end{tabular}

Table 2: Bin sizes, average kinematic values and results of the asymmetry amplitudes presented in Fig. 目.

\begin{tabular}{|c|c|c|c|c|c|c|c|c|}
\hline \multicolumn{3}{|c|}{ kinematic bin } & \multirow{2}{*}{$\begin{array}{c}\langle-t\rangle \\
{\left[\mathrm{GeV}^{2}\right]}\end{array}$} & \multirow{2}{*}{$\begin{array}{l}\left\langle x_{\mathrm{B}}\right\rangle \\
\overline{0.06}\end{array}$} & \multirow{2}{*}{$\begin{array}{c}\left\langle Q^{2}\right\rangle \\
{\left[\mathrm{GeV}^{2}\right]} \\
1.47\end{array}$} & \multirow{2}{*}{$\begin{array}{c}A_{\mathrm{LU}, \mathrm{I}}^{\sin \phi} \\
\pm \delta \text { (stat.) } \pm \delta \text { (syst.) } \\
.295 \pm 0.073 \pm 0.016\end{array}$} & \multirow{2}{*}{$\begin{array}{c}A_{\mathrm{LU}, \mathrm{DVCS}}^{\sin \phi} \\
\pm \delta \text { (stat.) } \pm \delta \text { (syst.) } \\
0.201 \pm 0.072 \pm 0.004\end{array}$} & \multirow{2}{*}{$\begin{array}{c}\begin{array}{c}A_{\mathrm{LU}, \mathrm{I}}^{\sin (2 \phi)} \\
\pm \delta \text { (stat.) } \pm \delta \text { (syst.) }\end{array} \\
0.015 \pm 0.071 \pm 0.008\end{array}$} \\
\hline & $\stackrel{\infty}{0}$ & $00-$ & & & & & & \\
\hline & $\dot{0}$ & $0.03-0.06$ & -0.04 & 0.06 & 1.56 & $-0.158 \pm 0.089 \pm 0.010$ & $0.019 \pm 0.089 \pm 0.017$ & $0.056 \pm 0.085 \pm 0.010$ \\
\hline & ต & $0.06-0.10$ & -0.08 & .06 & 1.55 & 22 & $-0.096 \pm 0$ & $-0.081 \pm 0.106 \pm$ \\
\hline & $\checkmark$ & $0.10-0.20$ & -0.14 & 0.06 & 1.57 & $-0.206 \pm 0.095 \pm 0.027$ & $-0.074 \pm 0.095 \pm 0.010$ & $0.046 \pm 0.093 \pm 0.014$ \\
\hline & 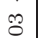 & $0.20-0.35$ & -0.26 & 0.06 & 1.69 & $-0.362 \pm 0$ & $0.280 \pm$ & $-0.101 \pm 0$ \\
\hline & $\dot{0}$ & $0.35-0.70$ & -0.46 & 0.05 & 1.78 & $-0.050 \pm 0$ & -0.1 & $-0.218 \pm c$ \\
\hline & $\mathcal{I}$ & $0.00-0.03$ & -0.02 & 0.09 & 2.32 & $-0.022 \pm 0.129 \pm 0.010$ & $-0.177 \pm 0.128 \pm 0.016$ & $0.095 \pm 0$. \\
\hline & $\dot{0}$ & $0.03-0.06$ & -0.04 & 0.10 & 2.50 & $-0.269 \pm 0.110 \pm$ & $0.086 \pm 0.1$ & $-0.180 \pm 0.105 \pm$ \\
\hline & & $0.06-0.10$ & -0.08 & 0.10 & 2.43 & $-0.254 \pm 0.125 \pm 0.018$ & $-0.088 \pm 0.123 \pm 0$ & $0.005 \pm 0.124 \pm$ \\
\hline & $\overline{8}$ & $0.10-0.20$ & -0.14 & 0.10 & 2.51 & $-0.258 \pm 0.107 \pm 0.015$ & $0.119 \pm 0$ & $0.001 \pm 0.106 \pm 0.028$ \\
\hline & 0 & $0.20-$ & -0 . & 0.10 & 2.74 & 025 & -0.0 & -0.0 \\
\hline & $\dot{0}$ & $0.35-0.70$ & -0.47 & 0.10 & 3.2 & $-0.062 \pm 0.242 \pm 0.026$ & $-0.060 \pm 0.242 \pm 0.026$ & $0.140 \pm 0.280 \pm 0.045$ \\
\hline & के & $0.00-0.03$ & -0.03 & 0.13 & 2.91 & $-0.037 \pm 0.368 \pm 0.046$ & $-0.271 \pm 0.370 \pm 0.104$ & $0.514 \pm 0.363 \pm 0.083$ \\
\hline & $V$ & $0.03-0.06$ & -0.05 & 0.15 & 3.62 & $-0.255 \pm 0.145 \pm 0.042$ & $0.238 \pm 0.145 \pm 0.027$ & $-0.073 \pm 0.150 \pm 0.048$ \\
\hline & 9 & $0.06-0.10$ & -0.08 & 0.16 & 3.93 & $-0.223 \pm 0.137 \pm 0.035$ & $0.059 \pm 0.137 \pm 0.00$ & $-0.099 \pm 0.135 \pm 0.008$ \\
\hline & 8 & $0.10-0.20$ & -0.14 & 0.17 & 4.30 & $-0.241 \pm 0.109 \pm 0.018$ & $0.023 \pm 0.108 \pm 0.012$ & $-0.208 \pm 0.108 \pm 0.018$ \\
\hline & ก & & -0.26 & 0.18 & 4.7 & $-0.242 \pm 0.135 \pm 0.039$ & $0.158 \pm 0.134 \pm 0.018$ & $-0.068 \pm 0.130 \pm 0.033$ \\
\hline & $\dot{0}$ & $0.35-0.70$ & -0.46 & 0.19 & 5.52 & $-0.284 \pm 0.188 \pm 0.034$ & $-0.187 \pm 0.185 \pm 0.028$ & $-0.284 \pm 0.175 \pm 0.035$ \\
\hline
\end{tabular}

Table 3: Bin sizes, average kinematic values and results of the asymmetry amplitudes presented in Fig. 3 . 


\begin{tabular}{|c|c|c|c|c|c|c|c|c|}
\hline \multicolumn{2}{|c|}{ kinematic bin } & \multirow{2}{*}{$\begin{array}{c}\langle-t\rangle \\
{\left[\mathrm{GeV}^{2}\right]} \\
-0.12 \\
\end{array}$} & \multirow{2}{*}{$\begin{array}{l}\left\langle x_{\mathrm{B}}\right\rangle \\
0.10 \\
\end{array}$} & \multirow{2}{*}{$\begin{array}{r}\left\langle Q^{2}\right\rangle \\
{\left[\mathrm{GeV}^{2}\right]} \\
2.46 \\
\end{array}$} & \multirow{2}{*}{$\begin{array}{c}A_{\mathrm{C}}^{\cos (0 \phi)} \\
\pm \delta \text { (stat.) } \pm \delta \text { (syst.) } \\
0.020 \pm 0.006 \pm 0.008\end{array}$} & \multirow{2}{*}{$\begin{array}{c}A_{\mathrm{C}}^{\cos \phi} \\
\pm \delta \text { (stat.) } \pm \delta \text { (syst.) } \\
0.055 \pm 0.009 \pm 0.004\end{array}$} & $\begin{array}{c}A_{\mathrm{C}}^{\cos (2 \phi)} \\
\pm \delta \text { (stat. }) \pm \delta \text { (syst.) }\end{array}$ & $\begin{array}{c}A_{\mathrm{C}}^{\cos (3 \phi)} \\
\pm \delta \text { (stat.) } \pm \delta \text { (syst.) }\end{array}$ \\
\hline & overall & & & & & & $-0.002 \pm 0.009 \pm 0.013$ & $-0.004 \pm 0.009 \pm 0.006$ \\
\hline \multirow{6}{*}{ 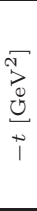 } & $0.00-0.03$ & -0.02 & 0.07 & 1.71 & $-0.027 \pm 0.014 \pm 0.009$ & $0.018 \pm 0.020 \pm 0.004$ & $0.008 \pm 0.020 \pm 0.008$ & $0.010 \pm 0.020 \pm 0.001$ \\
\hline & $0.03-0.06$ & -0.04 & 0.09 & 2.22 & $0.001 \pm 0.014 \pm 0.003$ & $-0.007 \pm 0.020 \pm 0.004$ & $-0.004 \pm 0.020 \pm 0.013$ & $0.004 \pm 0.020 \pm 0.005$ \\
\hline & $0.06-0.10$ & -0.08 & 0.10 & 2.44 & $0.003 \pm 0.015 \pm 0.011$ & $0.022 \pm 0.022 \pm 0.011$ & $0.022 \pm 0.022 \pm 0.013$ & $-0.015 \pm 0.022 \pm 0.009$ \\
\hline & $0.10-0.20$ & -0.14 & 0.11 & 2.72 & $-0.018 \pm 0.013 \pm 0.013$ & $0.067 \pm 0.018 \pm 0.012$ & $-0.038 \pm 0.018 \pm 0.021$ & $-0.019 \pm 0.018 \pm 0.004$ \\
\hline & $0.20-0.35$ & -0.26 & 0.12 & 3.13 & $-0.034 \pm 0.018 \pm 0.006$ & $0.160 \pm 0.025 \pm 0.019$ & $0.018 \pm 0.025 \pm 0.042$ & $-0.005 \pm 0.025 \pm 0.015$ \\
\hline & $0.35-0.70$ & -0.46 & 0.12 & 3.63 & $-0.056 \pm 0.029 \pm 0.009$ & $0.235 \pm 0.043 \pm 0.051$ & $0.041 \pm 0.040 \pm 0.025$ & $0.037 \pm 0.038 \pm 0.020$ \\
\hline \multirow{6}{*}{$\overbrace{i}^{\infty}$} & $0.03-0.06$ & -0.10 & 0.05 & 1.34 & $-0.043 \pm 0.014 \pm 0.014$ & $0.035 \pm 0.021 \pm 0.011$ & $0.004 \pm 0.019 \pm 0.007$ & $0.040 \pm 0.018 \pm 0.003$ \\
\hline & $0.06-0.08$ & -0.09 & 0.07 & 1.78 & $-0.014 \pm 0.013 \pm 0.007$ & $0.043 \pm 0.019 \pm 0.012$ & $-0.046 \pm 0.019 \pm 0.012$ & $-0.026 \pm 0.019 \pm 0.001$ \\
\hline & $0.08-0.10$ & -0.11 & 0.09 & 2.30 & $-0.048 \pm 0.016 \pm 0.014$ & $0.064 \pm 0.022 \pm 0.024$ & $0.033 \pm 0.022 \pm 0.019$ & $-0.005 \pm 0.022 \pm 0.011$ \\
\hline & $0.10-0.13$ & -0.12 & 0.11 & 2.92 & $0.010 \pm 0.017 \pm 0.009$ & $0.018 \pm 0.024 \pm 0.002$ & $0.024 \pm 0.023 \pm 0.004$ & $-0.035 \pm 0.023 \pm 0.005$ \\
\hline & $0.13-0.20$ & -0.16 & 0.16 & 4.04 & $-0.012 \pm 0.018 \pm 0.012$ & $0.088 \pm 0.025 \pm 0.032$ & $0.007 \pm 0.025 \pm 0.001$ & $-0.006 \pm 0.024 \pm 0.009$ \\
\hline & $0.20-0.35$ & -0.23 & 0.24 & 6.11 & $0.040 \pm 0.032 \pm 0.029$ & $0.041 \pm 0.045 \pm 0.014$ & $0.014 \pm 0.045 \pm 0.026$ & $-0.076 \pm 0.044 \pm 0.014$ \\
\hline \multirow{6}{*}{ 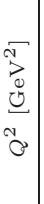 } & $1.0-1.4$ & -0.08 & 0.05 & 1.20 & $-0.041 \pm 0.013 \pm 0.021$ & $0.048 \pm 0.018 \pm 0.031$ & $0.018 \pm 0.018 \pm 0.010$ & $0.046 \pm 0.018 \pm 0.006$ \\
\hline & $1.4-1.8$ & -0.10 & 0.07 & 1.59 & $-0.033 \pm 0.015 \pm 0.020$ & $0.063 \pm 0.021 \pm 0.015$ & $-0.035 \pm 0.021 \pm 0.016$ & $-0.027 \pm 0.021 \pm 0.004$ \\
\hline & $1.8-2.4$ & -0.11 & 0.08 & 2.08 & $-0.012 \pm 0.015 \pm 0.014$ & $0.049 \pm 0.020 \pm 0.013$ & $-0.023 \pm 0.021 \pm 0.005$ & $-0.023 \pm 0.020 \pm 0.011$ \\
\hline & $2.4-3.2$ & -0.13 & 0.10 & 2.77 & $-0.025 \pm 0.016 \pm 0.006$ & $0.050 \pm 0.023 \pm 0.008$ & $0.021 \pm 0.022 \pm 0.019$ & $-0.001 \pm 0.023 \pm 0.014$ \\
\hline & $3.2-4.5$ & -0.15 & 0.13 & 3.76 & $0.021 \pm 0.018 \pm 0.009$ & $0.050 \pm 0.025 \pm 0.002$ & $-0.009 \pm 0.025 \pm 0.015$ & $-0.051 \pm 0.025 \pm 0.001$ \\
\hline & $4.5-10$ & -0.22 & 0.20 & 5.75 & $-0.001 \pm 0.021 \pm 0.014$ & $0.053 \pm 0.030 \pm 0.054$ & $0.029 \pm 0.030 \pm 0.006$ & $-0.002 \pm 0.030 \pm 0.011$ \\
\hline
\end{tabular}

Table 4: Bin sizes, average kinematic values and results of the asymmetry amplitudes presented in Fig. 团.

\begin{tabular}{|c|c|c|c|c|c|c|c|c|c|}
\hline \multicolumn{3}{|c|}{ kinematic bin } & \multirow{2}{*}{\begin{tabular}{|c}
$\begin{array}{c}\langle-t\rangle \\
{\left[\mathrm{GeV}^{2}\right]}\end{array}$ \\
-0.02
\end{tabular}} & \multirow{2}{*}{$\begin{array}{l}\left\langle x_{\mathrm{B}}\right\rangle \\
0.06\end{array}$} & \multirow{2}{*}{$\begin{array}{c}\begin{array}{c}\left\langle Q^{2}\right\rangle \\
{\left[\mathrm{GeV}^{2}\right]}\end{array} \\
1.47\end{array}$} & \multirow{2}{*}{$\begin{array}{c}A_{\mathrm{C}}^{\cos (0 \phi)} \\
\pm \delta \text { (stat.) } \pm \delta \text { (syst.) } \\
0.026 \pm 0.017 \pm 0.016\end{array}$} & \multirow{2}{*}{$\begin{array}{c}A_{\mathrm{C}}^{\cos \phi} \\
\pm \delta \text { (stat.) } \pm \delta \text { (syst.) }\end{array}$} & $\begin{array}{c}A_{\mathrm{C}}^{\cos (2 \phi)} \\
\pm \delta \text { (stat. }) \pm \delta \text { (syst. })\end{array}$ & \multirow{2}{*}{$\begin{array}{c}A_{\mathrm{C}}^{\cos (3 \phi)} \\
\pm \delta \text { (stat.) } \pm \delta \text { (syst.) } \\
\end{array}$} \\
\hline & $\stackrel{\infty}{\infty}$ & $0.00-0.03$ & & & & & & $-0.017 \pm 0.023 \pm 0.012$ & \\
\hline & & $0.03-0.06$ & -0.04 & 0.06 & 1.56 & $0.011 \pm 0.021 \pm 0.012$ & $0.032 \pm 0.028 \pm 0.006$ & $-0.036 \pm 0.029 \pm 0.008$ & $-0.004 \pm 0.029 \pm 0.002$ \\
\hline & 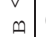 & $0.06-0.10$ & -0.08 & 0.06 & 1.55 & $-0.008 \pm 0.024 \pm 0.028$ & $0.007 \pm 0.034 \pm 0.007$ & $0.024 \pm 0.033 \pm 0.020$ & $0.025 \pm 0.033 \pm 0.025$ \\
\hline & & $0.10-0.20$ & -0.14 & 0.06 & 1.57 & $-0.041 \pm 0.022 \pm 0.034$ & $0.050 \pm 0.032 \pm 0.006$ & $-0.051 \pm 0.031 \pm 0.023$ & $0.002 \pm 0.030 \pm 0.006$ \\
\hline & $\dddot{q}$ & $0.20-0.35$ & -0.26 & 0.06 & 1.69 & $0.006 \pm 0.049 \pm 0.022$ & $0.241 \pm 0.084 \pm 0.033$ & $0.094 \pm 0.069 \pm 0.051$ & $0.095 \pm 0.052 \pm 0.025$ \\
\hline & 0 & $0.35-0.70$ & -0.46 & 0.05 & 1.78 & $0.196 \pm 0.196 \pm 0.068$ & $0.710 \pm 0.328 \pm 0.134$ & $0.302 \pm 0.242 \pm 0.060$ & $0.146 \pm 0.126 \pm 0.035$ \\
\hline & $\stackrel{N}{\sim}$ & $0.00-0.03$ & -0.02 & 0.09 & 2.32 & $-0.021 \pm 0.029 \pm 0.021$ & $0.065 \pm 0.042 \pm 0.039$ & $0.066 \pm 0.041 \pm 0.010$ & $0.028 \pm 0.040 \pm 0.018$ \\
\hline & $\dot{0}$ & $0.03-0.06$ & -0.04 & 0.10 & 2.50 & $-0.001 \pm 0.025 \pm 0.010$ & $-0.041 \pm 0.037 \pm 0.004$ & $0.062 \pm 0.036 \pm 0.020$ & $0.032 \pm 0.035 \pm 0.014$ \\
\hline & $\varphi$ & $0.06-0.10$ & -0.08 & 0.10 & 2.43 & $0.033 \pm 0.028 \pm 0.021$ & $0.050 \pm 0.039 \pm 0.006$ & $0.043 \pm 0.039 \pm 0.014$ & $-0.003 \pm 0.040 \pm 0.004$ \\
\hline & $\begin{array}{l}\overline{8} \\
\mathrm{~V}\end{array}$ & $0.10-0.20$ & -0.14 & 0.10 & 2.51 & $-0.022 \pm 0.025 \pm 0.026$ & $0.073 \pm 0.035 \pm 0.016$ & $-0.036 \pm 0.035 \pm 0.016$ & $-0.051 \pm 0.034 \pm 0.005$ \\
\hline & $\infty$ & $0.20-0.35$ & -0.26 & 0.10 & 2.74 & $-0.045 \pm 0.035 \pm 0.037$ & $0.170 \pm 0.049 \pm 0.009$ & $0.009 \pm 0.048 \pm 0.023$ & $-0.079 \pm 0.049 \pm 0.021$ \\
\hline & $\dot{0}$ & $0.35-0.70$ & -0.47 & 0.10 & 3.25 & $-0.118 \pm 0.081 \pm 0.020$ & $0.137 \pm 0.137 \pm 0.051$ & $0.041 \pm 0.117 \pm 0.024$ & $-0.047 \pm 0.101 \pm 0.021$ \\
\hline & $\stackrel{120}{20}$ & $0.00-0.03$ & -0.03 & 0.13 & 2.91 & $-0.181 \pm 0.081 \pm 0.028$ & $0.297 \pm 0.126 \pm 0.060$ & $0.161 \pm 0.110 \pm 0.046$ & $-0.010 \pm 0.118 \pm 0.016$ \\
\hline & & $0.03-0.06$ & -0.05 & 0.15 & 3.62 & $0.029 \pm 0.036 \pm 0.016$ & $-0.104 \pm 0.054 \pm 0.016$ & $0.014 \pm 0.051 \pm 0.011$ & $-0.075 \pm 0.049 \pm 0.005$ \\
\hline & 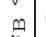 & $0.06-0.10$ & -0.08 & 0.16 & 3.93 & $-0.006 \pm 0.032 \pm 0.004$ & $-0.000 \pm 0.045 \pm 0.056$ & $0.014 \pm 0.046 \pm 0.006$ & $-0.101 \pm 0.044 \pm 0.008$ \\
\hline & \& & $0.10-0.20$ & -0.14 & 0.17 & 4.30 & $0.021 \pm 0.025 \pm 0.020$ & $0.041 \pm 0.036 \pm 0.017$ & $-0.007 \pm 0.035 \pm 0.015$ & $-0.036 \pm 0.035 \pm 0.006$ \\
\hline & $\stackrel{v}{\sim}$ & $0.20-0.35$ & -0.26 & 0.18 & 4.76 & $-0.015 \pm 0.031 \pm 0.004$ & $0.136 \pm 0.043 \pm 0.027$ & $0.038 \pm 0.042 \pm 0.013$ & $-0.022 \pm 0.042 \pm 0.008$ \\
\hline & : & $0.35-0.70$ & -0.46 & 0.19 & 5.52 & $-0.026 \pm 0.042 \pm 0.031$ & $0.199 \pm 0.059 \pm 0.017$ & $0.030 \pm 0.057 \pm 0.034$ & $0.095 \pm 0.057 \pm 0.063$ \\
\hline
\end{tabular}

Table 5: Bin sizes, average kinematic values and results of the asymmetry amplitudes presented in Fig. 司.

\section{References}

[1] D. Müller et al., Fortschr. Phys. 42 (1994) 101.

[2] X. Ji, Phys. Rev. Lett. 78 (1997) 610. X. Ji, Phys. Rev. D55 (1997) 7114.

[3] A.V. Radyushkin, Phys. Lett. B380 (1996) 417. A.V. Radyushkin, Phys. Rev. D56 (1997) 5524.

[4] M. Burkardt, Phys. Rev. D62 (2000) 071503; Erratum-ibid. D66 (2002) 119903. 
[5] A.V. Belitsky and D. Müller, Nucl. Phys. A711 (2002) 118.

[6] J.P. Ralston and B. Pire, Phys. Rev. D66 (2002) 111501.

[7] J. Blümlein, B. Geyer and D. Robaschik, Nucl. Phys. B560 (1999) 283.

[8] A.V. Belitsky and D. Müller, Nucl. Phys. B537 (1999) 397.

[9] A.V. Belitsky, A. Freund and D. Müller, Nucl. Phys. B574 (2000) 347.

[10] A.V. Belitsky and D. Müller, Phys. Lett. B486 (2000) 369.

[11] M. Diehl et al., Phys. Lett. B411 (1997) 193.

[12] A.V. Belitsky, D. Müller and A. Kirchner, Nucl. Phys. B629 (2002) 323.

[13] A.V. Belitsky and D. Müller, Phys. Lett. B417 (1998) 129.

[14] X. Ji and J. Osborne, Phys. Rev. D58 (1998) 094018.

[15] L. Mankiewicz et al., Phys. Lett. B425 (1998) 186.

[16] S. Wandzura and F. Wilczek, Phys. Lett. B72 (1977) 195.

[17] A.V. Belitsky and D. Müller, Nucl. Phys. B589 (2000) 611.

[18] N. Kivel and L. Mankiewicz, Eur. Phys. J. C21 (2001) 621.

[19] HERMES Collaboration, A. Airapetian et al., Phys. Rev. Lett. 87 (2001) 182001.

[20] CLAS Collaboration, S. Stepanyan et al., Phys. Rev. Lett. 87 (2001) 182002.

[21] CLAS Collaboration, X. Girod et al., Phys. Rev. Lett. 100 (2008) 162002.

[22] CLAS Collaboration, G. Gavalian et al., arXiv:0812.2950 [hep-ex].

[23] F. Ellinghaus (HERMES Collaboration), Proceedings for the Ringberg Workshop on New Trends in HERA Physics 2005, Ringberg Castle, Tegernsee, Germany, 2-7 Oct 2005. Published in "Ringberg 2005, New trends in HERA physics" 57-67. Edited by G. Grindhammer, B.A. Kniehl, G. Kramer, W. Ochs. World Scientific, 2006.

[24] HERMES Collaboration, A. Airapetian et al., Phys. Rev. D75 (2007) 011103.

[25] HERMES Collaboration, A. Airapetian et al., JHEP 06 (2008) 066.

[26] HERMES Collaboration, K. Ackerstaff et al., Nucl. Instr. and Meth. A417 (1998) 230.

[27] F. Ellinghaus, Ph.D. thesis, Humboldt Universität Berlin, Germany, January 2004, DESY-THESIS-2004-005.

[28] V.A. Korotkov and W.-D. Nowak, Eur. Phys. J. C23 (2002) 455.

[29] R. Barlow, Nucl. Instr. and Meth. A297 (1990) 496.

[30] M. Vanderhaeghen, P.A.M. Guichon and M. Guidal, Phys. Rev. D60 (1999) 094017.

[31] A. Vandenbroucke, Ph.D. thesis, Universiteit Gent, Belgium, November 2006, DESY-THESIS-2007-003.

[32] HERMES Collaboration, A. Airapetian et al., Phys. Lett. B648 (2007) 164.

[33] V. Guzey and T. Teckentrup, Phys. Rev. D74 (2006) 054027.

[34] V. Guzey and T. Teckentrup, Phys. Rev. D79 (2009) 017501. 
[35] F. Ellinghaus (HERMES Collaboration), Proceedings of the Workshop on "Exclusive Reactions at High Momentum Transfer", Jefferson Lab, Newport News, USA, May 2007, arXiv:0710.5768 [hep-ex].

[36] D. Zeiler, Ph.D. thesis, Universität Erlangen-Nürnberg, Germany, October 2009.

[37] A.V. Afanasev, M.I. Konchatnij and N.P. Merenkov, J. Exp. Theor. Phys. 102 (2006) 220.

[38] K. Goeke, M.V. Polyakov and M. Vanderhaeghen, Prog. Part. Nucl. Phys. 47 (2001) 401.

[39] M.V. Polyakov and C. Weiss, Phys. Rev. D60 (1999) 114017.

[40] A.V. Radyushkin, Phys. Rev. D59 (1999) 014030.

[41] A.V. Radyushkin, Phys. Lett. B449 (1999) 81.

[42] I.V. Musatov and A.V. Radyushkin, Phys. Rev. D61 (2000) 074027.

[43] M. Vanderhaeghen, P.A.M. Guichon and M. Guidal, Computer code for the calculation of DVCS and BH processes, Private Communication, 2001.

[44] M.V. Polyakov and A.G. Shuvaev, hep-ph/0207153.

[45] P.A.M. Guichon, L. Mosse and M. Vanderhaeghen, Phys. Rev. D68 (2003) 034018.

[46] K. Kumericki, D. Müller and K. Passek-Kumericki, Nucl. Phys. B794 (2008) 244.

[47] M. Guidal, Eur. Phys. J. A37 (2008) 319.

[48] K. Kumericki and D. Müller, arXiv:0904.0458 [hep-ph].

[49] H. Moutarde, Phys. Rev. D79 (2009) 094021.

[50] M. Guidal and H. Moutarde, arXiv:0905.1220 [hep-ph]. 